\title{
Mechanical Characterization and Modeling of Non-Linear Deformation and Fracture of a Fiber Reinforced Metal Matrix Composite
}

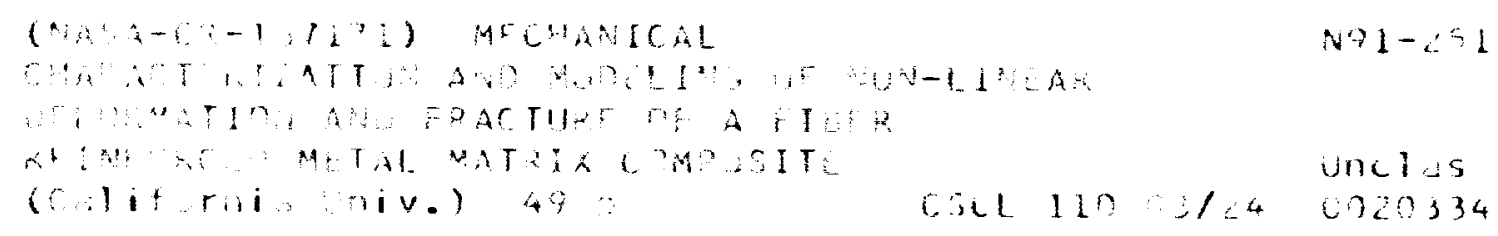

S. Jansson

University of California

Santa Barbara, California

June 1991

Prepared for

Lewis Research Center

Under Grant NAG3-894 



\title{
Mechanical Characterization and Modeling of Non-Linear Deformation and Fracture of a Fiber Reinforced Metal Matrix Composite
}

\author{
S. Jansson \\ University of California \\ Department of Mechanical and Environmental Engineering \\ Santa Barbara, California $\mathbf{4 3 1 0 6}$
}

\begin{abstract}
The non-linear anisotropic mechanical behavior of an aluminum alloy metal matrix composite reinforced with continuous alumina fibers has been determined experimentally. The mechanical behavior of the composite have been modeled by assuming that the composite has a periodical microstructure. The resulting unit cell problem has been solved with the finite element method. Excellent agreement was found between theoretically predicted and measured stress-strain responses for various tensile and shear loadings. The stress-strain responses for transverse and inplane shear were found to be identical and this will provide a simplification of the constitutive equations for the composite. The composite has a very low ductility in transverse tension and a limited ductility in transverse shear that has been correlated to high hydrostatic stresses that develop in the matrix. The shape of the initial yield surface has been calculated and good agreement was found between the calculated shape and the experimentally determined shape.
\end{abstract}




\section{INTRODUCTION}

Metal matrix composites are attractive because of high strength and stiffness to specific weight ratio and thermal stability. For example, they are considered in the automotive industry for replacing steel and aluminum in reciprocating and rotating engine components to reduce inertia forces, minimize vibrations, and increase allowable speed ranges. In suspension components to reduce the unsprung mass to improve handling. Metalmatrix composites have excellent properties when loaded in the fiber-direction but they will inevitably be subjected to transverse and shear loads when they are used in complex components, even if a substantial effort is made in the design to minimize these loadings. Transverse and shear loads are carried by the matrix and can set severe limitations on the load carrying capacity of composites. Successful use of these materials in complex components requires a rigorous understanding of the multiaxial behavior that is not limited to in-plane properties of panels. At the present time the composites are available in limited quantities and shapes and this limits the range of mechanical tests that can be performed to characterize the mechanical behavior and the experimental data that can be determined. This shortage of information can be overcome by developing models that can use the data from a limited number of tests to simulate the mechanical behavior of composites for more general loadings. The models can also be used to study the behavior of fictitious composites of different matrix and fiber combinations and the performance of the composite can be optimized for a specific application. They also provide the local stress and strain distribution in the composite for macroscopic loadings and global failure criteria can be derived for the composite based on failure criteria of the constituents.

The non-linear stress strain responses of an aluminum alloy metal matrix composite reinforced with continuous alumina fibers in a unidirectional lay-up have been determined experimentally for longitudinal and transverse tension and in-plane and transverse shear. All the strain components were measured to determine the multiaxial behavior of the 
composite. The non-linear deformation characteristics have been compared with numerically calculated responses for a model based on the assumption that the microstructure of the composite is periodic and the fibers are arranged in an hexagonal array. The shape of the initial yield surface has been calculated from the model and was compared with experimental data for various loadings. Compared to the matrix the composite has a low ductility for some of the matrix dominated failure modes. The local stress distributions in the composite have been calculated and the low ductility has been attributed to high hydrostatic stresses in the matrix.

\section{EXPERIMENTS}

\section{Composite Material}

The composite is Du Pont's FP/Al [Campion et al., 1978] with continuous fibers in a unidirectional lay-up. The fiber volume fraction was determined to be $55 \%$. The FP fiber consists of $99 \%$ pure crystalline $\alpha$-alumina $\left(\mathrm{Al}_{2} \mathrm{O}_{3}\right)$ coated with silica that improves the strength of the fiber and aids the wetting by the molten metal. The fibers have a diameter of approximately $20 \mu \mathrm{m}$, a modulus of 345 to $380 \mathrm{GPa}$, a tensile strength of 1.9 to $2.1 \mathrm{GPa}$ for $6.4 \mathrm{~mm}$ gauge length, and a fracture strain of $0.3-0.4 \%$. The matrix material is a $2 \mathrm{wt} \%$ Li-Al binary alloy. The lithium promotes the wetting of the alumina fibers that forms a strong matrix-fiber interface and it also raises the modulus and decreases the density of the matrix. The composite is fabricated by preparing the FP fibers into tapes by using a fugitive binder and the tapes are subsequently laid up in a metal mold in the desired orientation. The binder is burned away and the mold is vacuum-infiltrated with the molten matrix. The composite was available in the form of a plate $150 \times 150 \times 12.5 \mathrm{~mm}$ thick.

Specimens and Test Procedures

The specimen type used for longitudinal and transverse tests, Fig. 1., has a relatively large radius at the transition from the gripping section to the reduced gauge section to 
provide a low stress concentration and a short gauge length to prevent specimen buckling during compressive loading. The strains were measured with $3.2 \mathrm{~mm}$ strain gauges.

Shear properties were determined with specimens of Iosipescu-type, Fig 2, because the composite was available in the form of a plate. The specimen is a notched short beam in anti-symmetric loading with the symmetry line in the mid-span subjected to shear load and no bending moment. The notches provide a constant shear stress distribution along the symmetry line when the notch angle is selected appropriately [Wang and Dasgupta, 1986], $102.6^{\circ}$ for an isotropic material. However, the shear stress is singular at the notch tip if the notch is too sharp and it has a parabolic distribution along the symmetry line if the notch is too blunt. In practice, the notch angle is selected to be slightly larger than the critical, in order to be conservative and avoid high stress concentrations. The extent of the zone with constant shear strain in the longitudinal direction of the specimen decreases when the composite yields and short strain gauges are required to get an accurate measurement of the shear strain during the plastic deformation. The shear strain was measured with two 1.62 $\mathrm{mm}$ long strain gauges mounted on opposite sides of the specimen in the gauge section: one in $+45^{\circ}$ and one in the $-45^{\circ}$ direction. The reported shear stress has been calculated as the load divided by the cross sectional area of the gauge section. In-plane properties have been obtained for two fiber orientations: fibers orientated in the direction of the notches $(\alpha=\pi / 2$, $\beta=0$ in Fig. 2) and fibers orientated perpendicular to the notches $(\alpha=\pi / 2, \beta=\pi / 2)$.

A simply supported cylindrical plate subjected to a pressure load on one side, Fig. 3, was used to measure initial yield and limit strength for transverse bi-axial loading. The strain was measured with a $1.62 \mathrm{~mm}$ long strain gauge mounted in the center of the pressure free surfaces of the plate. Elementary plate theory proved to be sufficiently accurate to calculate the stresses at the surfaces at initial yield for the present dimensions of the plate. 
The initial yield surface was determined in a series of separate tests. The size of the initial yield surface is dependent on the definition of initial yield and the shape can be also strongly dependent on the definition of initial yield. Initial yield was defined as the point at which the stress strain curve has an offset strain of $10^{-5}$ to the initial linear part. This was the lowest limit that could be detected with good accuracy and repeatability for the test system. The loading was reversed at this point and the point on the opposite side of the yield surface was thereafter measured. The reported values represents the average value of five subsequent measurements on the same specimen.

All the specimens and fixtures were loaded in a servo-hydraulic test machine operated in displacement control. The loading rates used correspond to strain rates of the order $510^{-5} 1 / \mathrm{s}$. The specimens were machined to final dimensions by using diamond grinding.

\section{MECHANICAL PROPERTIES}

The measured moduli and Poisson's ratios are summarized in table 1. The notation is such that the fibers are orientated in the 3-direction and the transverse plane is the 1-2 plane. The Poisson's ratios have been determined form the tensile tests and the shear moduli from the shear tests. Five elastic constants are sufficient to describe the linear elastic response for a transversley isotropic material and they are interrelated through the relations

$$
\frac{v_{31}}{E_{33}}=\frac{v_{13}}{E_{11}} \quad G_{12}=\frac{E_{22}}{2\left(1+v_{12}\right)}
$$

The longitudinal stress strain curve, Fig. 4, is linear up to approximately $250 \mathrm{MPa}$, thereafter the matrix yields and the tangent stiffness decreases. A variation in the initial yield stress was observed between specimens indicating that the residual stress state in the 
matrix is affected by the handling of the material. The onset of yielding is accompanied with an increase in the transverse contraction, Fig. 5, that is caused by an increase in matrix contraction when the matrix yields. The fracture strain is of the same order as the reported fiber fracture strain, 0.3-0.4\% [Champion et. al., 1978] indicating that the final fracture is dominated by the fiber strength.

The transverse stress strain curve, Fig. 6, has a deviation from linearity at $75 \mathrm{MPa}$. The ultimate strength is $200 \mathrm{MPa}$ and the strain to fracture is $0.8 \%$. The ultimate strength is of the same magnitude as the matrix strength while the fracture strain of $0.8 \%$ is substantially lower than the matrix fracture strain of $30 \%$ [Sakui and Tamura, 1969]. The strain rate in the fiber direction, Fig. 7, decreases when the matrix yields and tends to zero for most of the tests. However some tests showed a final negative strain rate in the fiber direction. The magnitude of strain rate in the unloaded transverse direction, Fig. 8, increases when the matrix yields and the final slope is close to minus one indicating that the deformation tends to plane strain in the fiber direction. The increase in transverse contraction is caused by a continuous decrease in matrix tangent stiffness in the plastic deformation regime. During the plastic deformation the length change of the elastic fibers decreases and from the constant volume condition of the matrix deformation the ratio of $\dot{\varepsilon}_{11} / \dot{\varepsilon}_{22} \rightarrow-1$. The negative strain rate in the fiber direction at the end of some tests indicate that the matrix cannot always sustain the elastic shortening of the fibers and this may be caused by matrix damage that develops close to the transverse fracture.

Shear stress strain curves for strains up to $2 \%$ are shown in Fig. 9 for different loading directions. Inplane shear ( $\alpha=\pi / 2$ in Fig. 2) has been measured for two fiber orientations: fibers in the directions of the notches $(\beta=0)$ and in the longitudinal direction of the specimen $(\beta=\pi / 2)$. No systematic difference could be found between the stress strain curves for the two orientations. A deviation is not expected and this confirms that the 
test method is appropriate for the present composite. Transverse shear corresponds to $\alpha=0$ . The fibers are then orientated in the thickness direction of the specimen, and $\alpha=\pi / 4$ corresponds to shear loading with equal transverse and inplane shear stress components. The shear stress strain curves for all the different loading are approximately equal. The difference is well within the variation of composite properties. This implies that an enormous simplification can be made in the formulation of constitutive equations for the composite: the transverse and inplane shear responses can be assumed to be equal and the interaction for combinations of the two loadings is quadratic. The failure strain is dependent on the direction of the shear loading. For inplane shear the strain to fracture was estimated from the ram displacement to be $20 \%$. This is the same order of ductility as for the matrix. For shear loadings with a component of transverse shear $(\alpha \neq 0)$ the strain to fracture is approximately $5 \%$.

The transverse biaxial tensile strength was estimate to be $370 \mathrm{MPa}$ from the plate bending experiment Fig. 15 . The stress was calculated by using the perfectly plastic solution for a pressure loaded cylindrical plate following the $v$. Mises yield condition.

\section{NUMERICAL MODEL OF COMPOSITE MATERIAL}

The mechanical behavior of the composite has been calculated by use of the method of homogenization [Larsen, 1976, Len'e, 1986, and Jansson, 1990]. The method is based on the assumption that the composite has a periodic microstructure and that the wavelength of the global loading of the composite is much longer than the wavelength of the variation of the microstructure. In mechanical testing a relatively large material volume is subjected to a constant average field and the requirement of large difference in the two wavelengths is fulfilled everywhere except at the traction free surfaces. However this disturbance reaches approximately one unit cell into the composite and can be neglected. The great difference in wavelengths implies that only the two leading order terms of an asymptotic expansion of 
the displacement field in the two length scales need to be included and the global boundary value problem for the composite can be solved with an effective constitutive equation derived from a unit cell problem. Hence, the displacement field can be written as

$$
u_{i}=\varepsilon_{i j}^{o} x_{j}+u_{i}^{v}(x)
$$

where $\varepsilon_{i j}^{o}$ is the global average strain field in the composite that gives rise to a linear displacement field over the unit cell and

$$
u_{j}^{v}(x)=u_{j}^{v}\left(x_{k}+d_{k}\right)
$$

is a component of the displacement field that is periodic on the unit cell and is equal on opposite sides of the boundary of the unit cell of size $d_{k}$. The unit cell is the smallest repeating element in the composite and it is sufficient to define the spatial distribution of the constituents on the unit cell to define the distribution in the composite. The stresses in the unit cell are given by the constitutive equation for the constituents as

$$
\sigma_{\mathrm{ij}}=\mathrm{C}_{\mathrm{ijkl}}(\varepsilon, \mathrm{x}) \varepsilon_{\mathrm{kl}}
$$

where $C_{i j k l}(\varepsilon, x)$ is a function of position, given by the distribution of the constituents, and can be a nonlinear function of invariants of $\varepsilon$. The stress distribution in the unit cell must satisfy local equilibrium

$$
\sigma_{\mathrm{ij}, \mathrm{j}}=0
$$

on the unit cell. The equilibrium (4) equation together with the requirement of periodicity on the boundary on the unit cell of the unknown periodic displacement field $u_{j}^{v}(x)$ defines 
a well posed problem on the unit cell when $u_{j}^{v}(x)$ is fixed at some location. The average strain $\varepsilon_{\mathrm{ij}}^{\mathrm{o}}$ is given as input and the periodical component of the displacement field and the local stress distribution in the unit cell are solved for. The average stress in the unit cell can than be calculated from the local stress distribution as

$$
<\sigma_{i j}>=\frac{1}{v} \int_{v} \sigma_{i j}(x) d v
$$

In this way effective stress strain curves can be generated for the composite for different loadings and in addition the stress distribution $\sigma_{\mathrm{ji}}(\mathrm{x})$ gives local stress concentration factors.

The present composite consists of long fibers in a unidirectional lay up that are randomly distributed in the transverse plane. In the model to be analyzed the fibers are assumed to be long parallel cylinders arranged in a hexagonal array, Fig 10. This is the periodical array which has the mechanical properties with the closest symmetries to the properties of the composite with randomly distributed fibers. Both systems are transversely isotropic when the constituents are linear elastic but the hexagonal array has a weak deviation from transverse isotropy when the matrix exhibits a nonlinear stress strain relation [Jansson, 1990]. The deviation is most pronounce for a perfectly plastic matrix where some loadings permit slip on planes in the periodical arrays that are unconstrained by the fibers. This could not occur in a large volume element of a composite with randomly distributed fibers because it is not possible to find a straight line in the transverse plane that do not cut through fibers, cf. Underwood [1970]. Reasonable results can be expected if effective properties are calculated for loadings that do not permit slip on planes that are unconstrained by the fibers. The hexagonal array then resembles closely to a composite with randomly distributed fibers. For the present fiber volume fraction the shear loading 
$\tau_{13}$ permits slip on unconstrained planes and that response cannot be expected to be representative for a transversely isotropic composite.

In the analysis the fibers are assumed to be linear elastic and the matrix behavior is modeled with a small strain $\mathrm{J}_{2}$ deformation theory for a power law material. The total strain $\varepsilon_{\mathrm{ij}}$ is given as the sum of an elastic and a plastic part

$$
\varepsilon_{i j}=\varepsilon_{i j}^{e}+\varepsilon_{i j}^{p}
$$

where the elastic part is given as

$$
\varepsilon_{j i}^{e}=\frac{1+v}{E} \sigma_{i j}-\frac{v}{E} \sigma_{k k} \delta_{i j}
$$

and the plastic part is defined as

$$
\varepsilon_{\mathrm{ij}}^{\mathrm{p}}=\frac{3}{2}\left(\frac{1}{\mathrm{E}_{\mathrm{s}}}-\frac{1}{\mathrm{E}}\right) \mathrm{s}_{\mathrm{ij}}
$$

where $E$ is Young's modulus, $v$ the poisson's ratio, and $s_{i j}$ the stress deviator. The secant modulus is given as $\mathrm{E}_{\mathrm{s}}=\sigma_{\mathrm{e}} / \varepsilon_{\mathrm{e}}$ and has the same slope as the uniaxial stress strain curve. Here is $\sigma_{\mathrm{e}}$ the $\mathrm{v}$. Mises equivalent stress and $\varepsilon_{\mathrm{e}}$ is the associated equivalent strain. The stress strain relation reduces to

$$
\varepsilon=\left\{\begin{array}{cc}
\frac{\sigma}{\mathrm{E}} & \sigma<\sigma_{\mathrm{o}} \\
\sigma_{\mathrm{o}}\left(\frac{\sigma}{\sigma_{\mathrm{o}}}\right)^{\mathrm{n}} & \sigma \geq \sigma_{\mathrm{o}}
\end{array}\right\}
$$

in uniaxial tension, where $\sigma_{0}$ is the initial yield stress in uniaxial tension. 
The governing boundary value problem for the effective properties on the unit cell is two dimensional and because of the non-linearity and the complex geometry it has been solved with the help of the Finite Element Method. The displacement field is interpolated with nine nodes isoparametric elements and reduced integration is used to avoid locking, 2 $\times 2$ for the hydrostatic component and $3 \times 3$ for the deviatoric component of the stress tensor. The nonlinear system of equations is solved with a Newton Raphson scheme. All the considered loading of the unit cell, Fig. 10, are symmetric or anti-symmetric with respect to the $y_{1}$ and $y_{2}$ axis. The displacement field has an inversion symmetry at the point $(\sqrt{3} / 2 \mathrm{~b}, \mathrm{~b} / 2)$ when the displacements are taken to be zero at the point. This implies that only an eight of the indicated unit cell in Fig. 10 needs to be analyzed, Fig. 11. The finite element mesh in Fig. 11 was determined to be sufficiently fine to give convergent solutions for non-linear matrix behavior. A detailed description of the implementation of the method and derivations of the boundary conditions for different loadings are given in Jansson [1990].

\section{CALCULATED EFFECTIVE STRESS STRAIN RELATIONS}

The effective elastic constants of the composite have been calculated by using elastic constants from the literature for the constituents; fiber [Champion et. al., 1978 and Richerson, 1982] and matrix [Dudzinski, 1952 and Noble et al., 1982]. The calculated elastic constants are compared with the experimentally determined elastic constants in Table 1. The maximum difference between experimental and calculated values is less than $10 \%$ and this is well within the limit of how precise this type of calculations can be expected to be because of the uncertainty in the value of the elastic constants of the constituents. Other methods exist that could be used to calculate the individual elastic constants with a reasonable accuracy. However, the value of elastic constants calculated by combining results for different models can be very inaccurate [Jansson 1990]. The present method has the advantage of providing a consistent way to calculate all the elastic 
constants.

The elastic properties of fiber and matrix are not greatly affected by the history of processing and heat treatment of the composite and hence it is possible to use data from the literature. However, the flow properties of the $\mathrm{Al}-\mathrm{Li}$ matrix alloy are strongly dependent on heat treatment and cold-working [Stark et al., 1981 and Sakui and Tamura, 1969]. Details of the processing of the composite and of any heat treatments are not available. Hence, the exact state of the matrix is not known and the information is insufficient for finding the flow properties of the matrix from the literature. This leaves as the only means of estimating the flow properties of the matrix in the composite to fit a calculated response, by varying the flow properties of the matrix, to a matrix dominated stress-strain curve for the composite. After fitting the calculated response for loading in the 1-direction (Fig. 10) to the transverse stress strain curve, Fig. 6, the initial yield stress of the matrix was determined to $94 \mathrm{MPa}$ with a hardening exponent $\mathrm{n}=5$.

The applicability of the method can now be evaluated by examining how well it can predict non-linear effective stress strain response for loadings other than transverse tension and the ratio between the different strain components.

The calculated contraction in the fiber direction for transverse tension, Fig. 7, shows the same trend as the measured and tends asymptotically to plane strain in the fiber direction. The contraction in the other transverse direction shows also the same characteristics as the experiment and tends asymptotically to a slope of minus one. No damage is included in the constitutive equations describing the matrix behavior and the contraction in Fig. 7 indicating softening in the matrix close to fracture cannot be modeled with the present constitutive equation. 
The calculated response for longitudinal tension is compared with measured in Fig. 4. The overall agreement is good. However the calculation predicts that the initial non-linearity of the stress-strain curve occurs at stress higher than the experiments show. It was found from the measurements of the initial yield surface that the composite has a residual stress state such that the initial yield stress is lower in tension than in compression for longitudinal loading. The effect of residual stress from the fabrication of the composite has not been included in the calculations but would change the results in the direction indicated by the experiments. The calculated transverse contraction, Fig. 5, is slightly lower than the measured for large strains.

It was found by Jansson [1990 ] that the calculated shear response for transverse shear, $\tau_{12}$, orientations are given in Fig. 10, and the inplane shear, $\tau_{13}$, are nearly identical. The inplane shear response, $\tau_{23}$, has the same linear elastic response but a lower limit load. It was stated that the loading $\tau_{23}$ is not likely to simulate the behavior of a composite with randomly distributed fibers in the transverse plane because the loading admits slip on planes that are unconstrained by the fibers. It was also found that the inplane and transverse shear moduli are equal within $10 \%$ when the fibers are istropic for a wide range of moduli ratios and volume fractions.

The experiments, Fig. 9, also indicate that the inplane ( $\alpha=\pi / 2$, Fig. 2) and the transverse $(\alpha=0)$ shear responses are similar and can be assumed to be identical. A loading with a equal components of transverse and inplane shear shows also a similar response. The calculated response agrees well with the measured responses.

\section{INITIAL YIELD SURFACE}

In the calculations, initial yielding is defined as when the matrix initially yields. The different macroscopic stress states causing initial yielding form a surface in the stress space. 
The surface is useful for determining if a cyclic stress state will have elastic shakedown and may be used as a flow potential for the plastic deformation of the composite. The response to initial yielding is elastic and the initial yield surface can therefore be determined from calculations based on linear elastic constituents. It was shown by Jansson [1990] that the initial yield surface for a hexagonal array does not in general possess transverse isotropi for transverse loading and inplane shear. For example, the stress concentration of the v. Mises equivalent stress is higher for transverse tension in the 1-direction than in the 2-direction. Two approaches were suggested to overcome this discrepancy between the surfaces for the hexagonal array and the transversely isotropic material. The unit cells can be assumed to be randomly orientated with regard to the stress state. The size of the initial yield surface is then dictated by the unit cell that has the orientation that corresponds to the highest stress concentration. The surface is calculated for a given stress state by searching through all possible orientations of the unit cell for the lowest stress that causes initial yielding and a substantial amount of computations are needed to define the whole initial yield surface. A more attractive modification is to assume that the principal stresses in the transverse plane are always orientated in the $I$ and $I I$ directions shown in Fig. 10. These directions are orientated $15^{\circ}$ off the symmetry axis in the transverse plane and they are equivalent with the same stress concentration. The surface based on this definition has all the symmetry properties of a transversely isotropic material and is less cumbersome to calculate than the surface based on the highest stress concentration. The two surfaces do not differ greatly in shape [Jansson, 1990]. The definition that the principal stresses are always orientated in the I and II-direction is used here.

The calculated initial yield surface is a circle in the $\sigma_{13}-\sigma_{23}$ plane, Fig. 12, and has the shape of a rugby ball in the $\sigma_{11}-\sigma_{22}$ plane, Fig.13, with sharp corners at $\sigma_{11}=$ $\sigma_{22}$. Cuts through the surface in the $\sigma_{11}-\sigma_{22}-\sigma_{33}$ space are shown in Fig. 14. The surface has the form of a thin slab that is long in the $\sigma_{11}=\sigma_{22}$ and $\sigma_{33}$ directions and 
is thin in the direction corresponding to transverse shear $\sigma_{11}=-\sigma_{22}$.

The linear elastic stress distribution in the unit cell is governed by two uncoupled problems: an antiplane problem gives the two inplane shear stresses $\left(\sigma_{13}\right.$ and $\left.\sigma_{23}\right)$ and a plane problem gives the other stress components $\left(\sigma_{11}, \sigma_{22}, \sigma_{33}\right.$ and $\left.\sigma_{12}\right)$. The V. Mises equivalent stress is quadratic in the stress components and can be written in terms of the macroscopic stresses for a given location in the matrix as

$$
\sigma_{\mathrm{e}}^{2}=\mathrm{f}_{1}^{2}\left(\sigma_{11}, \sigma_{22}, \sigma_{33}, \sigma_{12}\right)++\mathrm{f}_{2}^{2}\left(\sigma_{13}, \sigma_{23}\right)
$$

where $f_{1}$ is given by the solution to the plane problem and $f_{2}$ is given by the solution to the antiplane problem. The initial yield surface is quadratic for this interaction when the possition for the highest stress concentrations does not varie with loading. The functions $f_{1}$ and $\mathrm{f}_{2}$ are then given by the surfaces in Figs. 12 and 14 respectively. In general the location of the highest stress concentration will vary and the assumption of a quadratic interaction will give a conservative estimate of the initial yield surface.

The experimentally determined points on the initial yield surface have been normalized with respect to the yield stress of the matrix, $94 \mathrm{MPa}$, and are also shown in Fig. 12-14. The data points correspond to key points defining the dimensions of the surfaces and supports the calculated shape. They are not sufficiently close in the stress space to give the detailed shape of the corners of the surface. This requires multiaxial tests in which the ratio between the stress components can be varied. The yield surface must be a circle in the $\sigma_{13}-\sigma_{23}$ plane because of the symmetry of the material and the inplane shear experiments gives the radius the circle, Fig.12. The calculated yield surface for transverse loading is given together with yield surfaces for the matrix subjected to plane stress and plane strain in Fig. 13. The fibers introduce stress concentrations that gives the composite a 
lower initial yield stress than the yield stress of the matrix for stress states close to uniaxial tension and shear $\left(\sigma_{11}=-\sigma_{22}\right)$. However, transverse bi-axial tension or compression loads the fibers in the longitudinal direction and causes hydrostatic stress to build up in the matrix that more than compensates for the stress concentration induced by the fibers and the composite has a higher initial yield stress for loadings close to biaxial tension than the matrix. The biaxial plate bending experiment, Fig. 3 and 15, verifies the result given by the calculations that the initial yield stress is substantially higher for bi-axial transverse tension than in shear and transverse tension. It is obvious for longitudinal tension that the fibers help to carry the loading and the initial yield stress must be higher than the yield stress of the matrix, Fig. 14. The experiments show that the size of the calculated yield surface and the measured in this direction are comparable. However, the experiments indicate that the initial yield stress is lower in tension than in compression. The ceramic fibers have a lower coefficient of thermal expansion than the metal matrix. During cool down, from the processing temperature of the composite, the missmatch will load the matrix in residual tension in the longitudinal direction. This residual stress state will cause the composite to initially yield for a lower stress in longitudinal tension then for longitudinal compression.

Measurements of the initial yield surface for mixed loading of a $\mathrm{B} / \mathrm{Al}$ system have been reported in [Dvorak and Bahei-El-Din, 1987]. The data points are not sufficient close in stress space to make strong statements about the detailed shape of the surface. However, it was advocate that the surface should be represented by a bimodal model. It appears as the model is more appropriate for describing the limit load behavior of the composite.

\section{COMPOSITE FRACTURE}

Longitudinal Tension

The strength in the longitudinal direction is dominated by the fiber strength. The applicability of some simple models to predict the composite strength based on the 
statistical variation of the fiber strength and the yield stress of the matrix were evaluated by Nunes [1982]. It was found that two models gave reasonable predictions. In the first model is it assumed that the composite strength is given by the fiber bundle strength and the flow stress of the matrix. Thus,

$$
\sigma_{11}^{f}=c_{f} \sigma_{F B}\left(l_{g}\right)+\left(1-c_{f}\right) \sigma_{y m}\left(\varepsilon_{f}\right)
$$

where $c_{f}$ is the fiber volume fraction, $\sigma_{y m}\left(\varepsilon_{f}\right)$ is the flow stress of the matrix at fracture, and $\sigma_{F B}\left(l_{g}\right)$ is the fiber bundle strength for the gauge length $l_{g}$ of the specimen. The relation between fiber bundle strength and average fiber strength is given by [Colman, 1958] as

$$
\sigma_{\mathrm{FB}}=\bar{\sigma}_{\mathrm{f}} \frac{\left[\mathrm{m} \text { e } \mathrm{l}_{\mathrm{g}} / 1_{\mathrm{o}}\right]^{\frac{1}{\mathrm{~m}}}}{\Gamma\left(\frac{\mathrm{m}+1}{\mathrm{~m}}\right)}
$$

where $\bar{\sigma}_{f}$ is the average strength of fibers of length $l_{o}, m$ is the Weibull modulus and $\Gamma$ is the gamma function. In the second model [Zweben and Rosen, 1970] it is assumed that global fracture occurs when a fiber adjacent to a previously broken fiber breaks because of the stress concentration induced by the initially broken fiber. This leads to the tensile strength

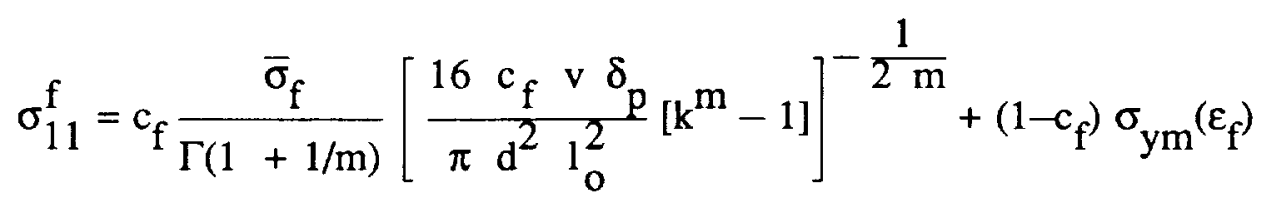

where $v$ is the loaded volume, $k$ the stress concentration in the fiber next to a broken fiber, $d$ is the fiber diameter, and $\delta_{p}$ is the pull out length of the fiber. The average 
strength of uncoated fibers is reported by Champion et. al [1978] to be $\bar{\sigma}_{\mathrm{f}}=1480 \mathrm{MPa}$ for a gauge length $1_{o}=6.25 \mathrm{~mm}$ and the Weibull modulus for the strength of the fibers $\mathrm{m}=$ 6.5 [Nunes, 1982]. The maximum pull out length was measured from the fracture surfaces of the longitudinal specimens and was found to be $\delta_{p} \approx 2 \mathrm{~d}$. The stress concentration factor $k$ is dependent on the arrangement of the fibers. The value for a broken fiber in a square array, $\mathrm{k}=1.15$, was used in the analysis and is the same value as used by Nunes [1982]. The matrix yield stress at fracture was estimated to $115 \mathrm{MPa}$. For this data the parallel model with the fiber bundle strength predicts $\sigma_{11}^{\mathrm{f}}=580 \mathrm{MPa}$ and the model with the local interaction between fibers predicts $\sigma_{11}^{\mathrm{f}}=512 \mathrm{MPa}$. The measured average strength was here found to be $585 \mathrm{MPa}$. The fiber bundle model gives here a better prediction that the local interaction model. This is the opposite to what was found by Nunes [1982]. It appears as if the local interaction model is more physically reasonable but its derivation is based on many simplifying assumptions that remain to be worked out in detail. The simplicity of the fiber bundle model and its accurate prediction makes it attractive.

\section{Transverse and Shear Fractures}

Inspection of the fracture surfaces for transverse tension and shear revealed that nearly all the the fibers at the fracture surface are covered by a metal matrix layer. This indicates that the interface between fiber and matrix is strong and that the transverse tensile and shear strengths are governed by the matrix strength.

The highest normal loading on the fiber matrix interface in transverse tension occurs for loading in the 2-direction, Fig 10. The initial elastic stress concentration is 1.4 for this loading, Fig. 16. The stress concentration subsequently decreases after the initial yielding of the matrix to the value 1.17 and it thereafter starts to increase with continued loading to a value close to the initial elastic stress concentration. 
The highest stress concentration at the interface for inplane shear occurs for the shear loading $\tau_{13}$. The initial elastic value is 1.36 , Fig. 16. The shear stress concentration also decreases after the initial yielding of the matrix but it never builds up to its initial value with subsequent loading.

It can be deduced that it is conservative to use the initial elastic stress concentration for estimating the loading on the interface. For the present fiber and matrix the elastic stress concentration for a single fiber in an infinite body [Goodier, 1933] is 1.33 for transverse tension and 1.68 for inplane shear. A comparison of these values with the values for the periodical array, Fig. 16, shows that the interaction between the fibers reduces the loading on the interface.

The experiments showed that the strain to failure is $0.8 \%$ for transverse tension, $5 \%$ for transverse shear, and $20 \%$ for inplane shear. The only stress components in inplane shear are $\sigma_{13}$ and $\sigma_{23}$. This implies that no hydrostatic stress is present in the matrix for this loading. However, transverse tension and transverse shear causes hydrostatic stress in the matrix. A measure of the constraint on the loading of the matrix is the void growth factor $\sigma_{\mathrm{kk}} / \sigma^{\mathrm{e}}$ where $\sigma_{\mathrm{kk}}$ is the sum of the principal stresses and $\sigma^{\mathrm{e}}$ is v. Mises equivalent stress [Rice and Tracy, 1969]. The strain to failure of a ductile material with small inclusions, that can act as sources for void nucleation, has been found to be strongly dependent on the void growth factor [Hancock and Mackenzie,1976] and this has been observed for an $\mathrm{Al}-\mathrm{Li}$ alloy by Pilling and Rindly [1986]. A stress state corresponding to a high void growth factor will cause a fast void growth and low strain to failure, caused by the coalesce of the voids, and conversely a low void growth factor will cause a slower void growth with a higher strain to failure.

The evolution and spatial distribution of the void growth factor in the matrix is 
depicted in Fig. 17 for transverse tension in the 1-direction and for transverse tension in the 2-direction in Fig. 18. The highest value of the void growth factor is approximately 2.5 at initial yield for both loading directions. It increases to 5 at $1 \%$ strain for loading in the 1-direction and to 7 for loading in the 2-direction. The location of the highest void growth factor is dependent on the loading direction. It is close to the symmetry axis for $\sigma_{22}$ loading and and is orientated off the symmetry axis for $\sigma_{11}$ loading. The extent of the zones with a high void growth factor is large for these loadings. The void growth factor at initial yielding for transverse shear, Fig. 19, is approximately equal to the value for transverse tension. It does not increase to such a high value as for transverse tension during the subsequent loading. Furthermore, the extent of the zone with a high void growth factor is much smaller than for transverse tension.

The low ductility in transverse tension can be correlated to high hydrostatic stresses that build up in the matrix when it deforms plastically for this loading. Transverse shear has lower hydrostatic stresses extending over smaller zones than for transverse tension and this indicates that the composite should have a higher ductility. This is in agreement with experiments that show a higher ductility for transverse shear than for transverse tension. The inplane shear does not cause any hydrostatic stress and the ductility should be high as indicated by the experiments. It can therefore be concluded that the matrix ductility should not be sensitive to hydrostatic stress for a composite with a strong fiber matrix interface if it is desirable to have a composite with high ductility in transverse tension.

\section{CONCLUSIONS}

It was found that all the elastic constants for a continuous fiber reinforced metal matrix composite can be calculated accurately from the properties of the constituents by assuming that the microstructure is periodic. 
The non-linear response of the metal matrix is not known but it was demonstrated that the matrix properties could be extracted from one test and that the calculated responses for other loadings agrees well with the experiments.

Good correlation was found between the calculated shape of the initial yield surface and the experimental measurements. However, the experiments indicate that a residual stress state exists such that the initial yield stress is lower in transverse tension than in transverse compression. This indicates a residual longitudinal tensile stress in the matrix and is consistent with a residual stress state induced during cool down after the consolidation by the thermal mismatch between fiber and matrix.

The composite has a low ductility in transverse tension that has been related to high hydrostatic stresses that build up in the matrix for this loading when the matrix deforms plastically.

The stress strain curves for inplane shear, transverse shear, and combination of these loadings are for all practical purposes identical for this composite that has a high fiber volume fraction $(55 \%)$. The shear responses are also identical for the pure matrix. This corresponds to the other extreme case of a very low volume fraction. It is very likely that the responses for intermediate volume fractions are close. This implies that the constitutive equations describing the non-linear behavior for a composite with a strong fiber matrix bond will have a less complex form.

\section{ACKNOWLEDGMENTS}

This work has been supported by Chrysler Corporation through Chrysler Challenge Funds to University of Illinois and a grant from the NASA Lewis Research Center to University of California at Santa Barbara. 


\section{REFERENCES}

Champion, A. R., Krueger, W. H., Hartman, H. S., and Dhingra, A. K., 1978, "Fiber FP Reinforced Metal Matrix Composites," in: Proceedings of the 2nd International Conference on Composite Materials, Toronto, Canada, April 1978, The Metallurgical Society of AIME, pp. 883-904.

Colman, B. D. 1958, "On the Strength of Classical Fibres and Fibre Bundles Strength," Journal of the Mechanics and Physics of Solids, vol. 7, pp. 60-70.

Dvorak, G. J., Bahei-El-Di, Y. A., 1987, "A Bimodal Plasticity Theory of Fibrous Composite Material," Acta Mechanica, vol. 69, pp. 219-241.

Dudzinski, N., 1952-53, " The Young's Modulus, Poisson's Ratio and Rigidity Modulus of Some Aluminum Alloys," Journal of the Institute of Metals, vol. 81, pp. $49-55$.

Goodier, 1933, "Concentration of Stress Around Spherical and Cylindrical Inclusions and Flaws," Journal of Applied Mechanics, vol. 1, pp. 39-44.

Hancock, J. W. and Mackenzie, A. C., 1976, "On the Mechanisms of Ductile Fracture in High strength Steels Subjected to Multiaxial Stress," Journal of The Mechanics and Physics of Solids, vol. 24, pp. 141-169.

Jansson, S., 1990, "Homogenized Nonlinear Constitutive Properties and Local Stress Concentrations for Composites with Periodical Internal Structure," Department of Mechanical Engineering, UC Santa Barbara. 
Larsen, E. W., 1976, "Neutron Transportation and Diffusion in Inhomogeneous Media. II," Nuclear Science and Engineering, vol. 60, pp. 443-458.

Lene, F., 1986. "Damage Constitutive Relations for Composite Materials," Engineering Fracture Mechanics, vol. 25, pp. 713-728.

Noble, B., Harris, S. J., and Dinsdale, K., 1982, "The Elastic Modulus of Alumina-Lithium Alloys," Journal of Materials Science, vol. 17, pp. 461-468.

Nunes, J., 1982, "Tensile Property Evaluation of Polycrystaline Alumina Filaments and Their Composites," AMMRC TR 82-61, Army Materials and Mechanics Research Center, Watertown, Ma.

Pilling, J. and Ridley, N., 1986, "Role of Hydrostatic Pressure on Cavitation During Superplastic Flow of Al-Li Alloy," in: Aluminum - Lithium Alloys III, The Institute of Metals, London, pp. 184-190.

Rice, J. R. and Tracy, D. M., 1969, "On the Ductile Enlargement of Voids in Triaxial Stress Fields," Journal of the Mechanics and Physics of Solids, vol. 17, pp. 201-217.

Richerson, D. W., 1982, "Modern Ceramic Engineering," Marcel Dekker Inc., New York.

Sakui, S. and Tamura, M., 1969, "Yielding Phenomena of an Al-3\% Li Alloy," Transaction of the Japan Institute of Metals, vol. 10, pp. 343-350. 
Shetty, H. R. and Chou T. W., 1985, "Mechanical Properties and Failure Characteristics of FP/Aluminum and W/Aluminum Composites," Metallurgical Transactions A, vol. 16A, pp. 853-864.

Starke, E. A., Sanders, T. H., and Palmer, I. G., 1981, "New Approaches to Alloy Development in the Al-Li Systems," Journal of Metals, vol. 33, pp. 24-32.

Underwood, E. E., 1970, "Quantitative Stereology," Addison-Wesley, Reading, Ma.

Wang, S. S. and Dasgupta, A., 1986, "Development of Iosipescu-Type Test for Determining In-plane Shear Properties of Fiber Composite Materials: Critical Analysis and Experiment," UILU-ENG-86-5021, Department of Theoretical and Applied Mechanics, UI at Urbana Champaign.

Zweben, C. and Rosen, B. W., 1970, "A Statistical Theory of Material Strength with Application to Composite Materials," Journal of the Mechanics and Physics of Solids, vol. 18, pp. 189-206. 
Table $1 \quad$ Elastic properties.

\begin{tabular}{llllllll}
\hline & $\mathrm{E}_{11}$ & $\mathrm{E}_{33}$ & $\mathrm{G}_{12}$ & $\mathrm{G}_{13}$ & $v_{12}$ & $v_{31}$ & $v_{13}$ \\
& $\mathrm{GPa}$ & $\mathrm{GPa}$ & $\mathrm{GPa}$ & $\mathrm{GPa}$ & & & \\
\hline Experiments & 150 & 225 & 55 & 58 & 0.31 & 0.28 & 0.18 \\
Calculated & 148 & 220 & 54.9 & 57.3 & 0.336 & 0.281 & 0.189 \\
& & & & & & & \\
& & & & & & \\
$\mathrm{E}_{\mathrm{f}}=344.5 \mathrm{GPa}$ & $(50 \mathrm{Msi})$ & & $v_{\mathrm{f}}=0.26$ & & & \\
$\mathrm{E}_{\mathrm{m}}=68.9 \mathrm{GPa} \quad(10 \mathrm{Msi})$ & & $v_{\mathrm{m}}=0.32$ & & & & \\
$\mathrm{c}_{\mathrm{f}}=55 \%$ & & & & & & &
\end{tabular}



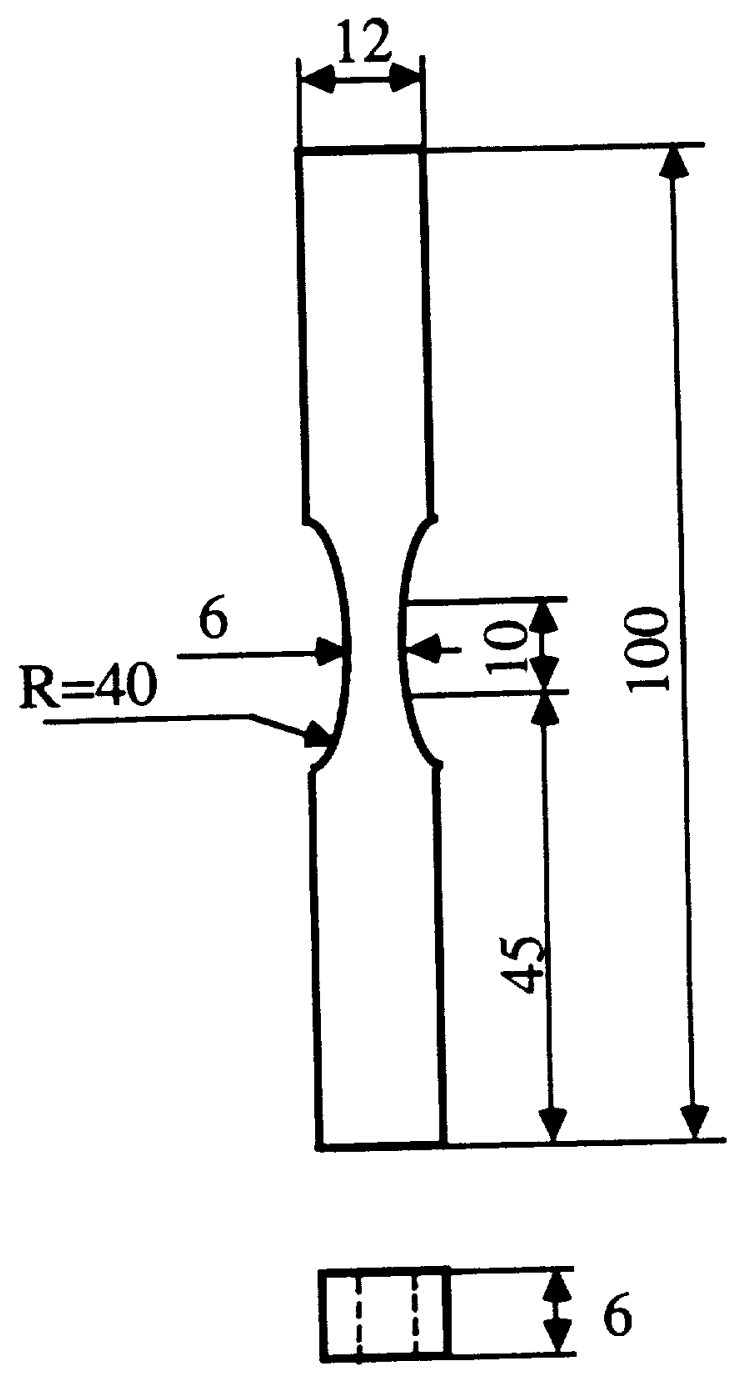

Fig. 1 Specimen used for longitudinal and transverse tests. 

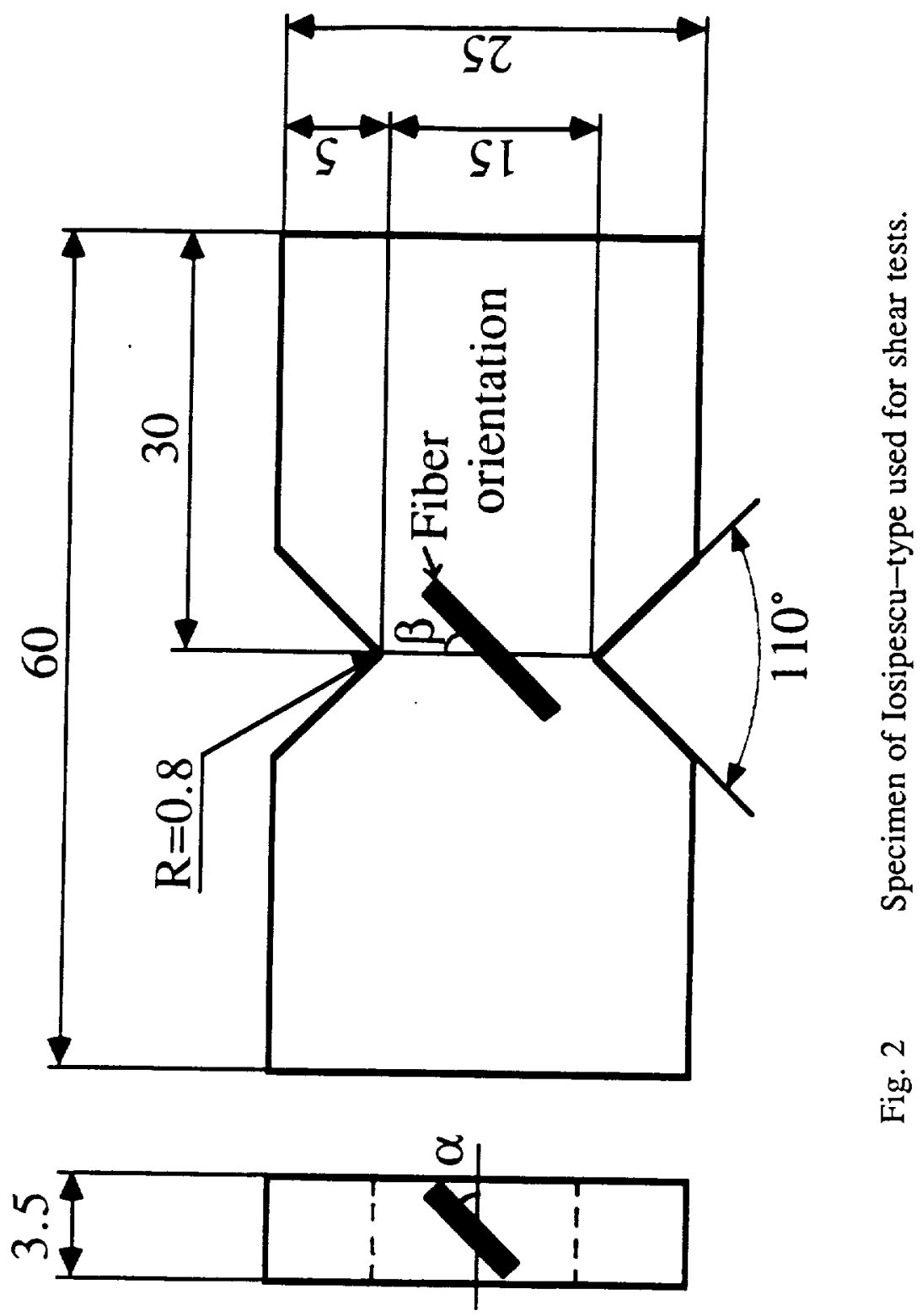


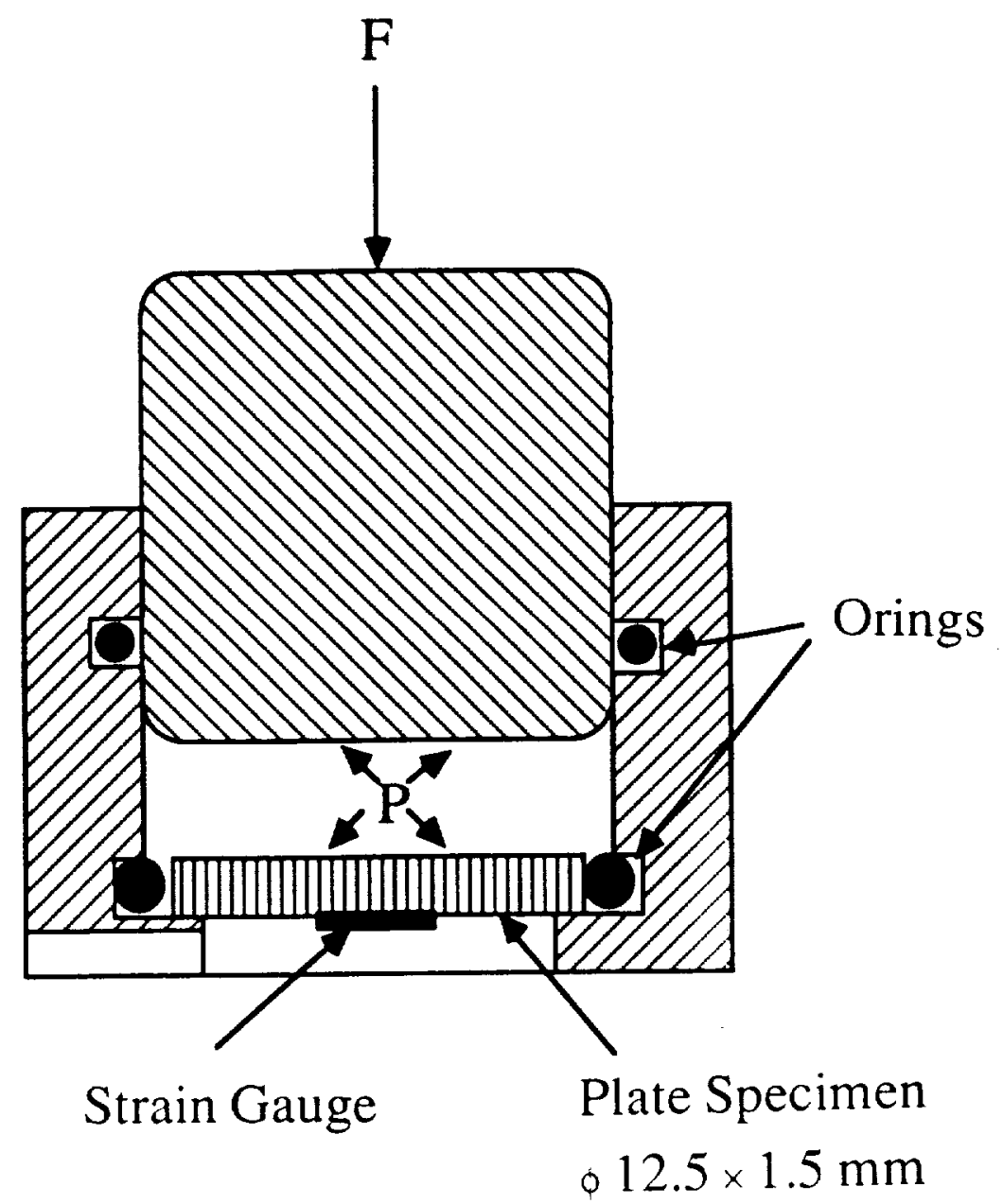

Fig. 3 Schematics of fixture used to measure initial yield surface for transverse biaxial tension. 


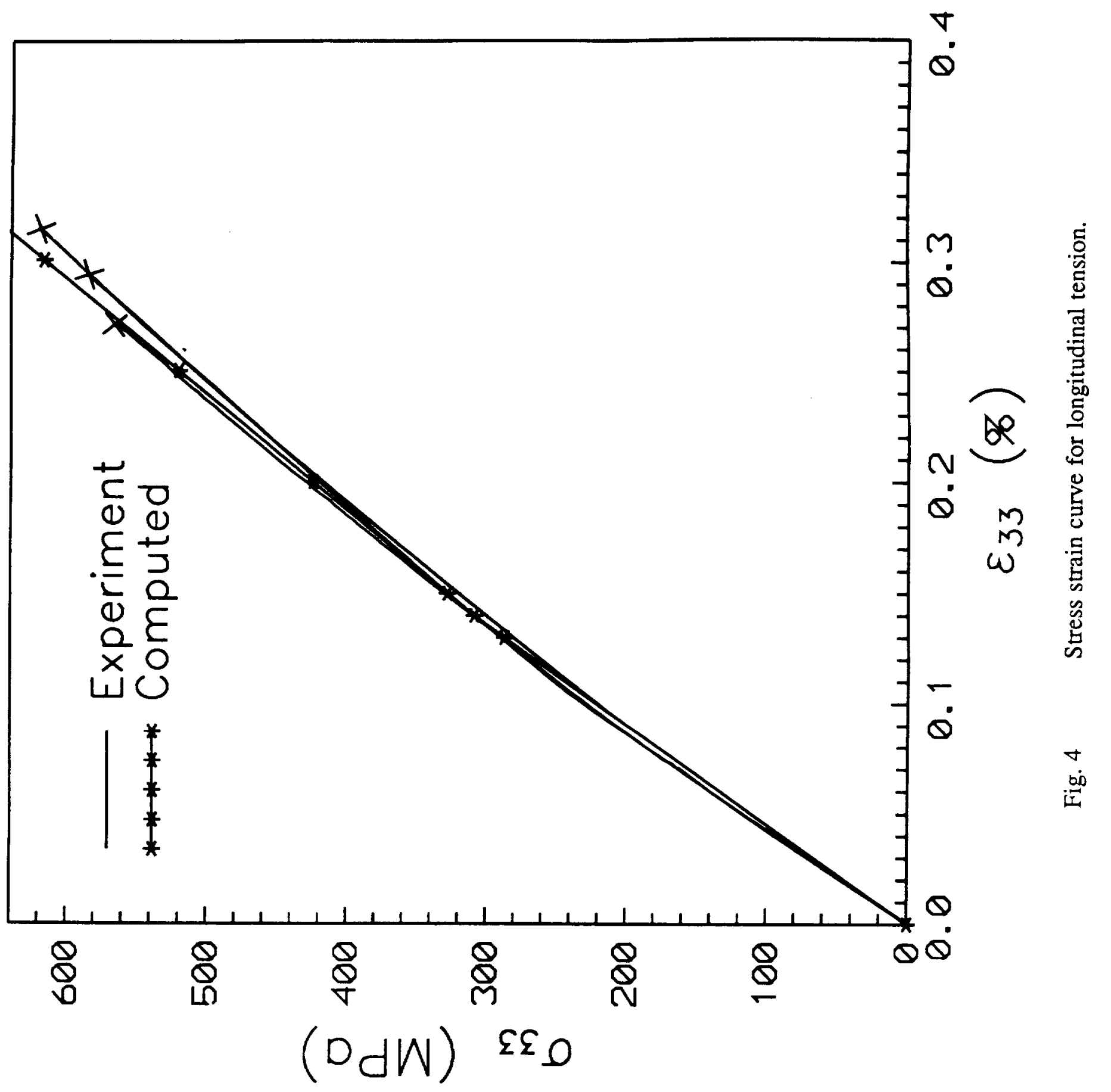




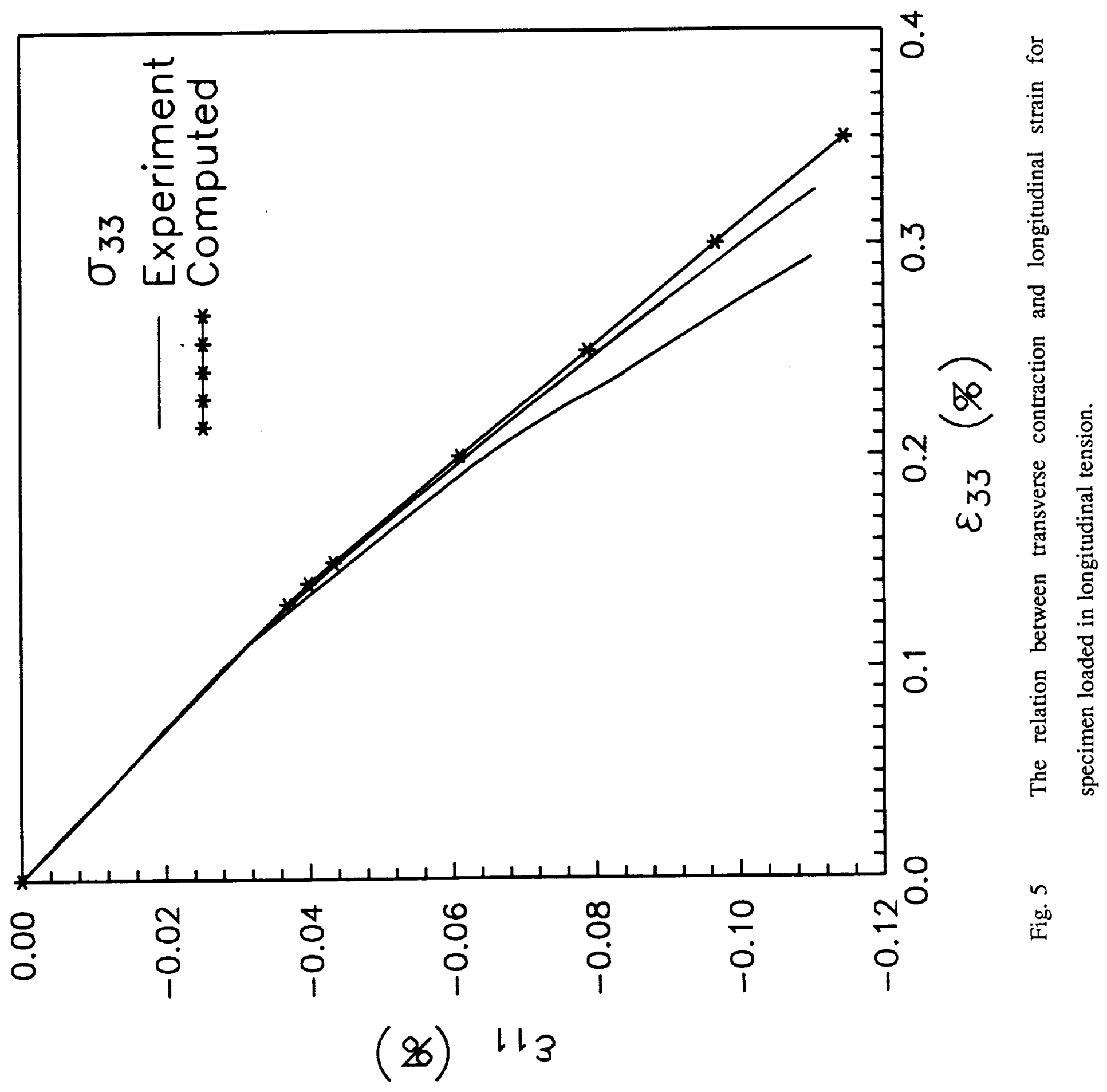




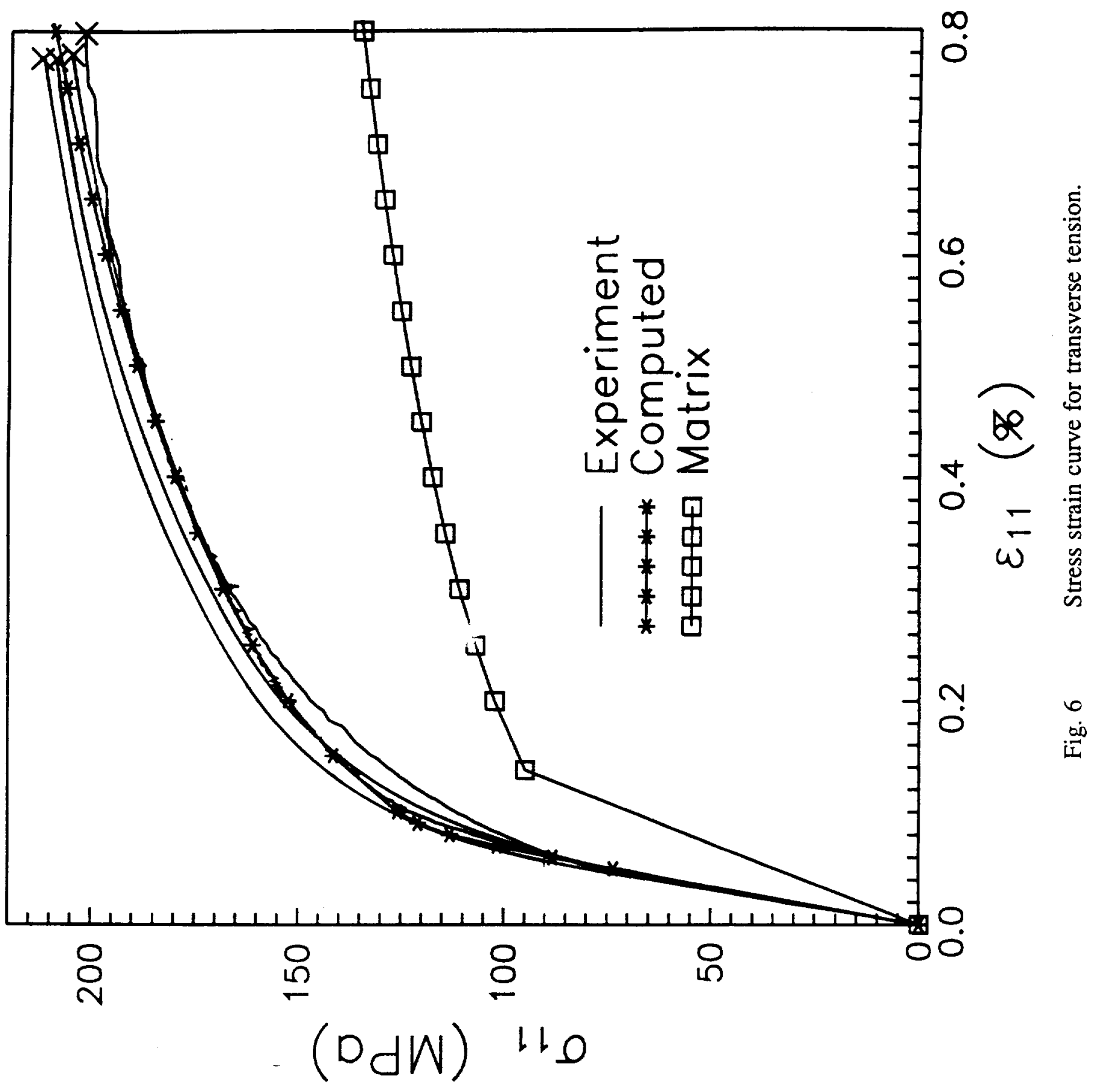




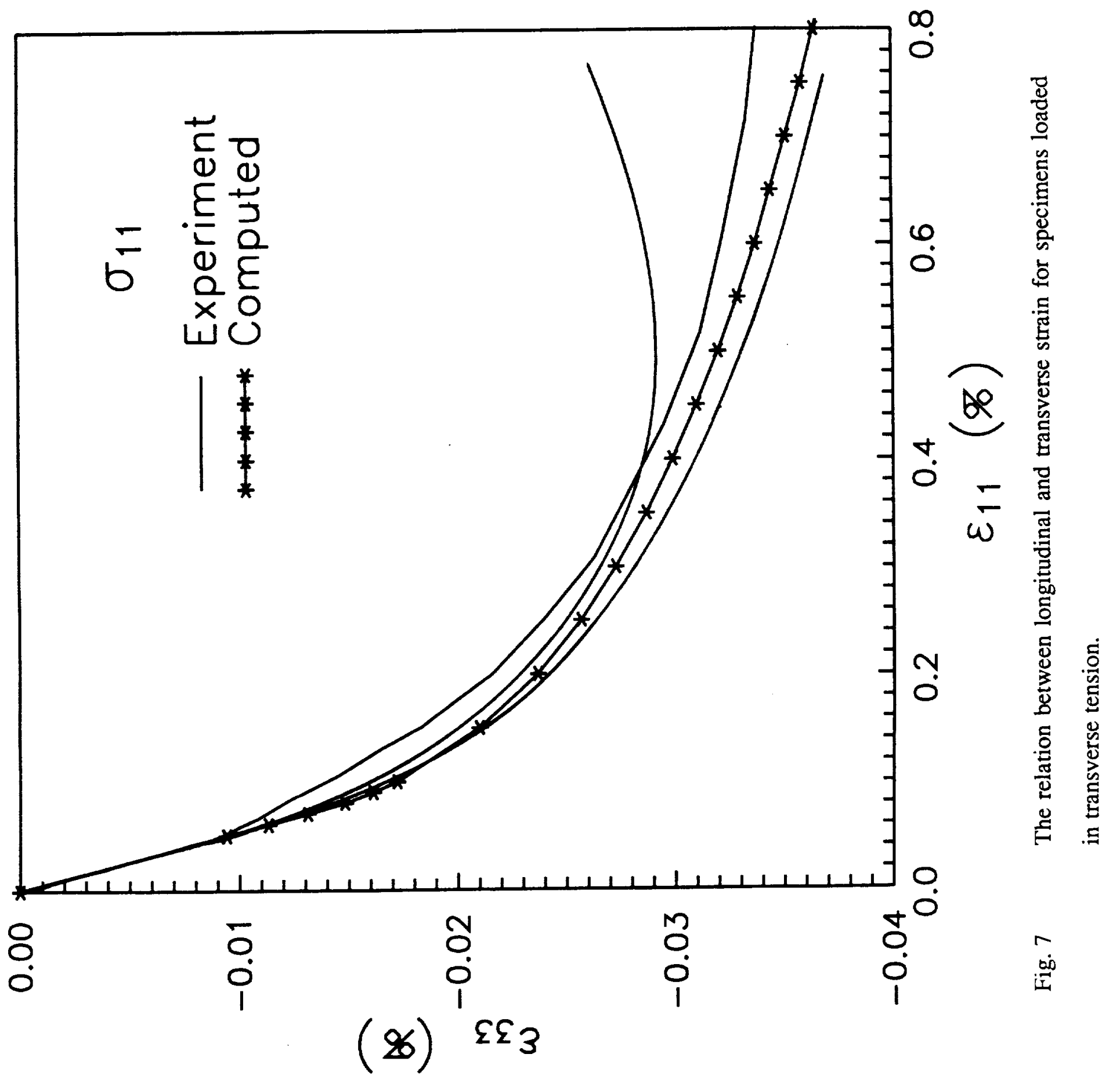




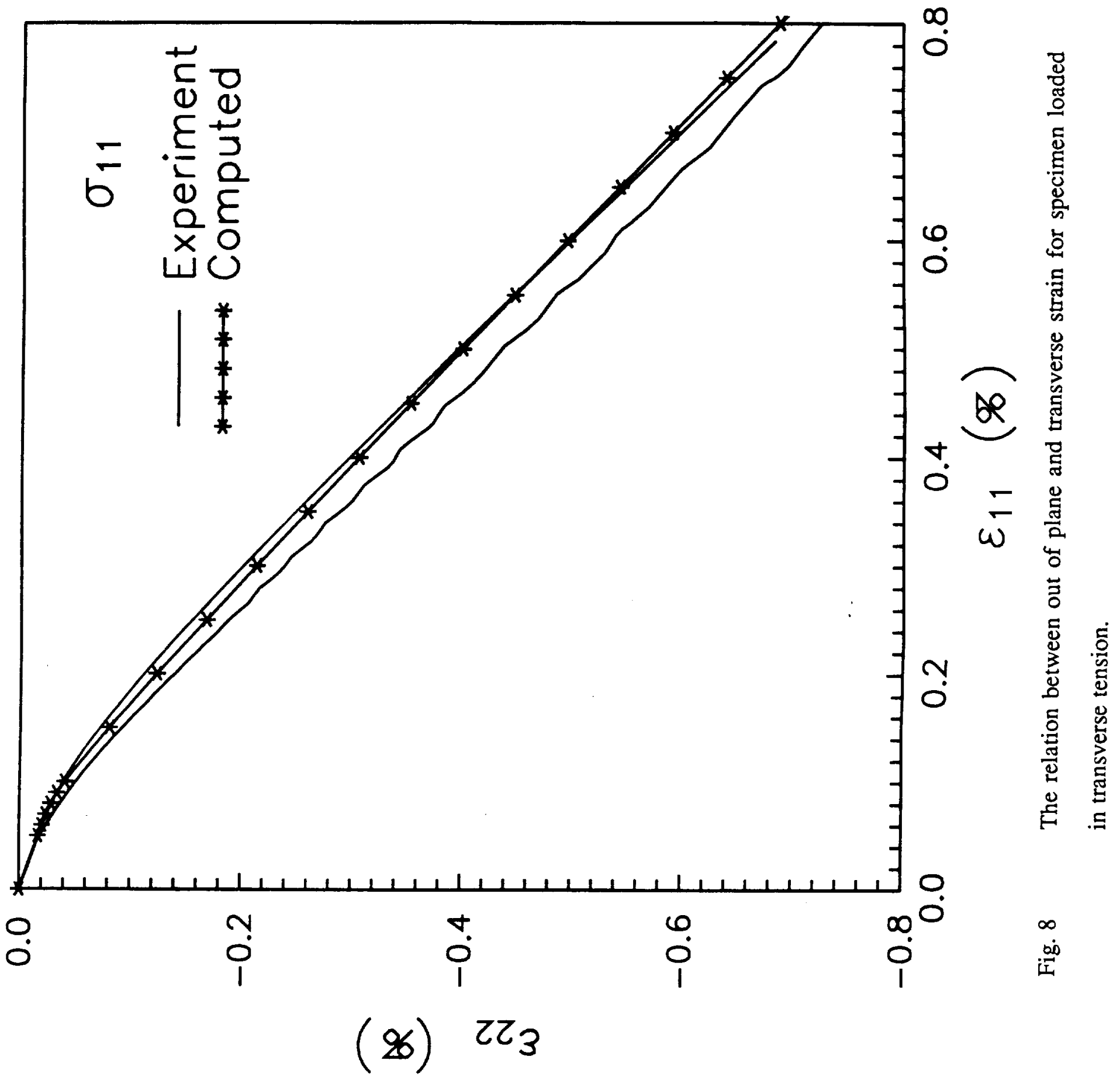




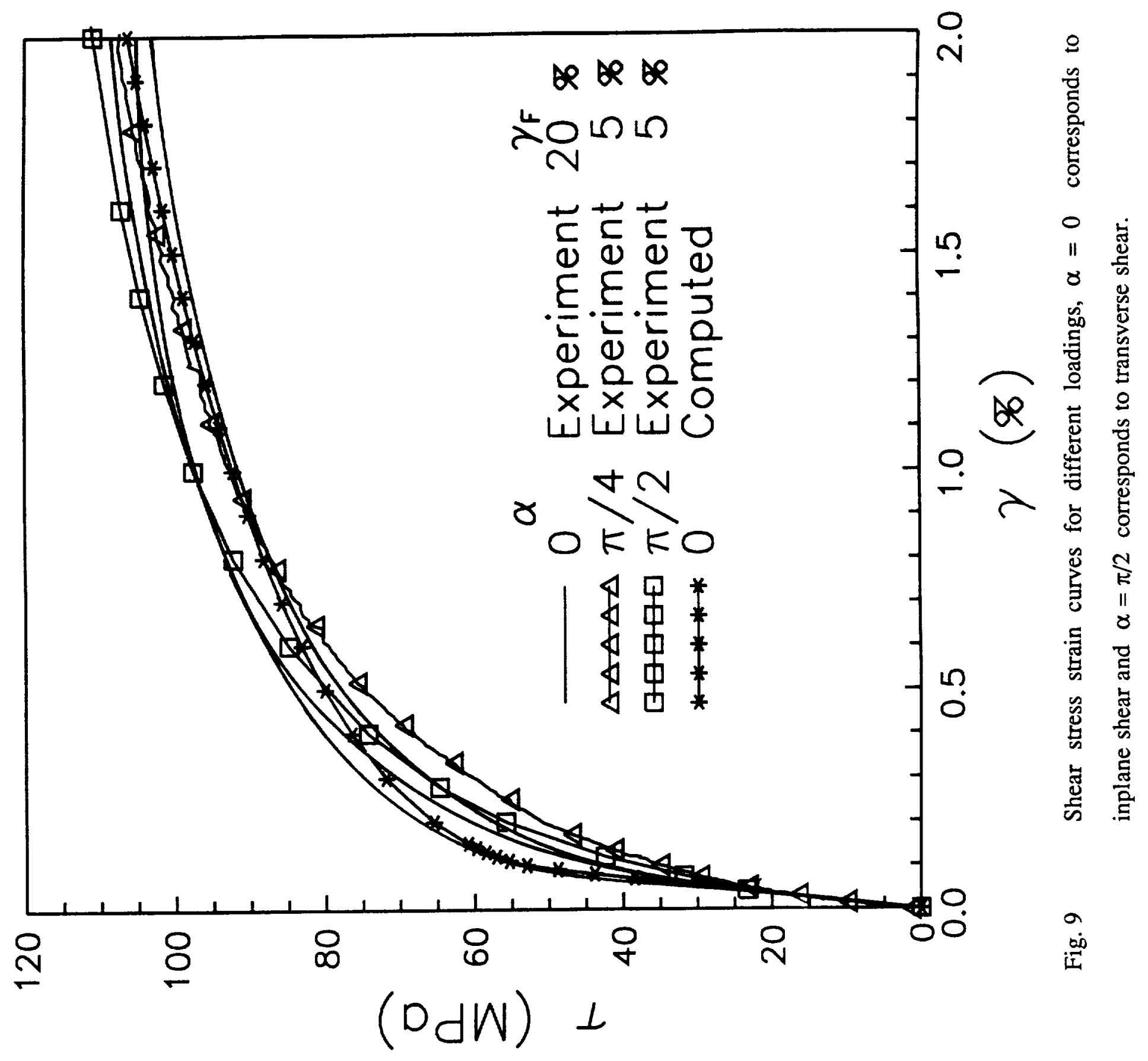




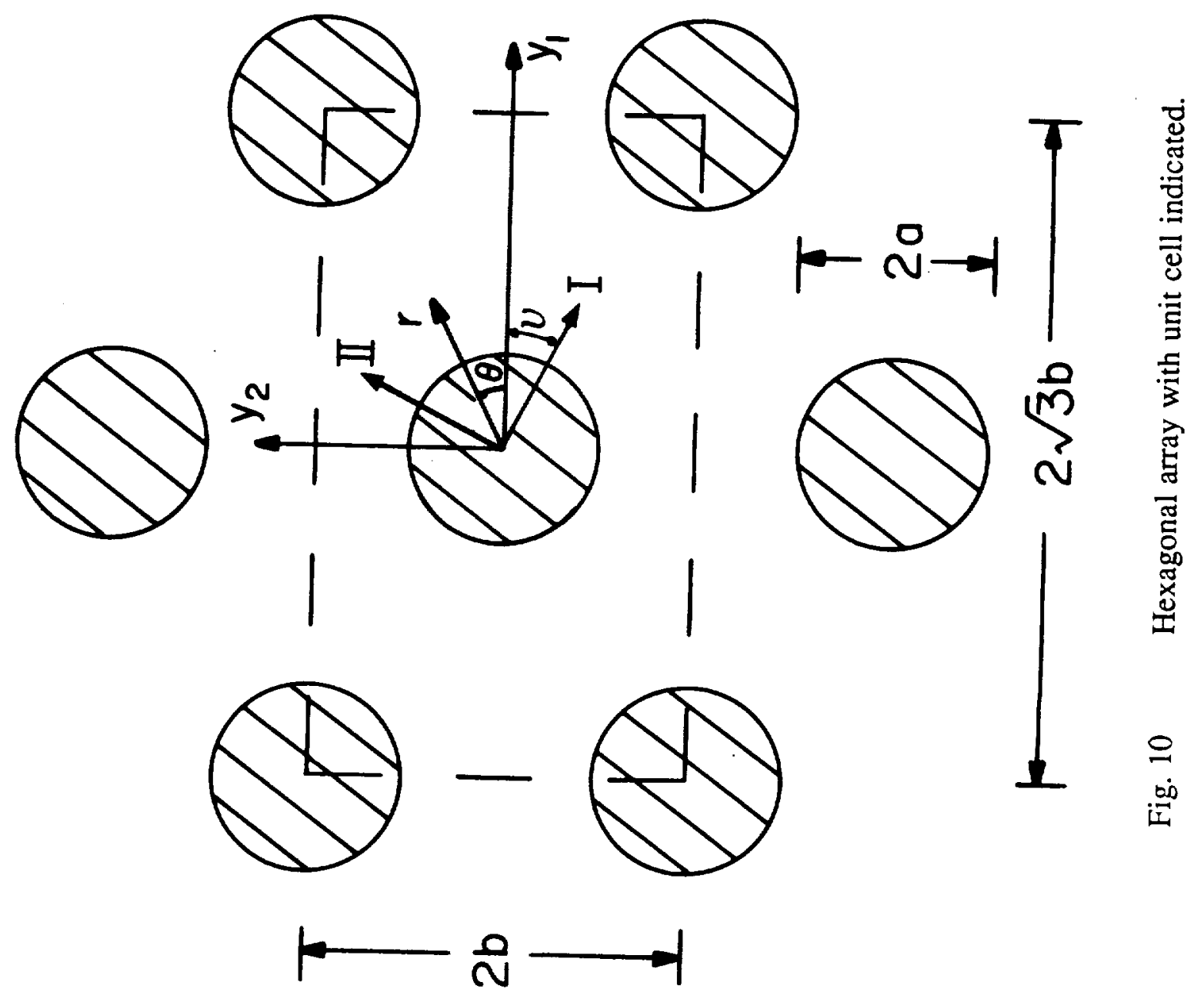




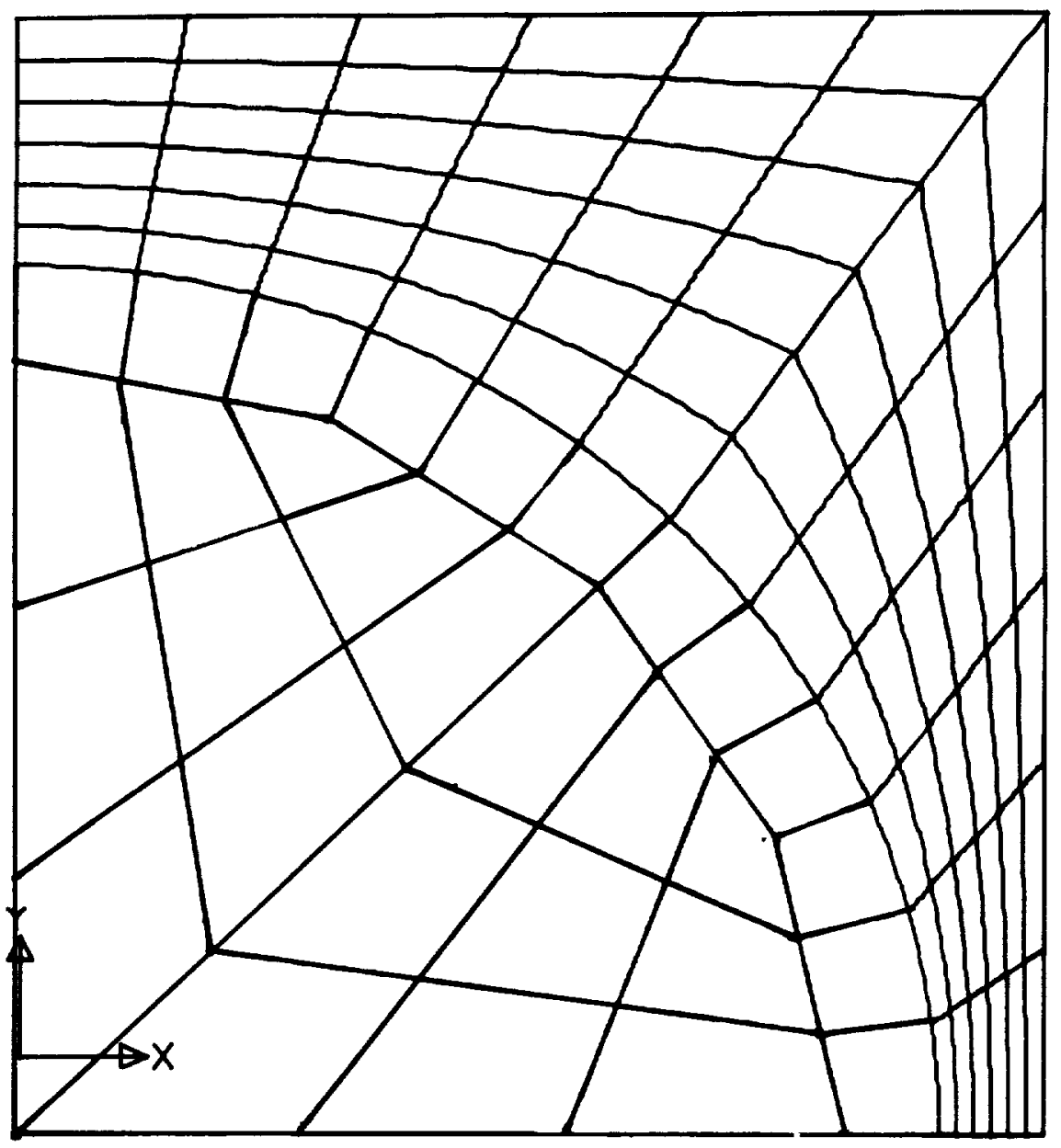

Fig. 11 Finite element mesh used in the calculations of effective properties and stress concentrations. 


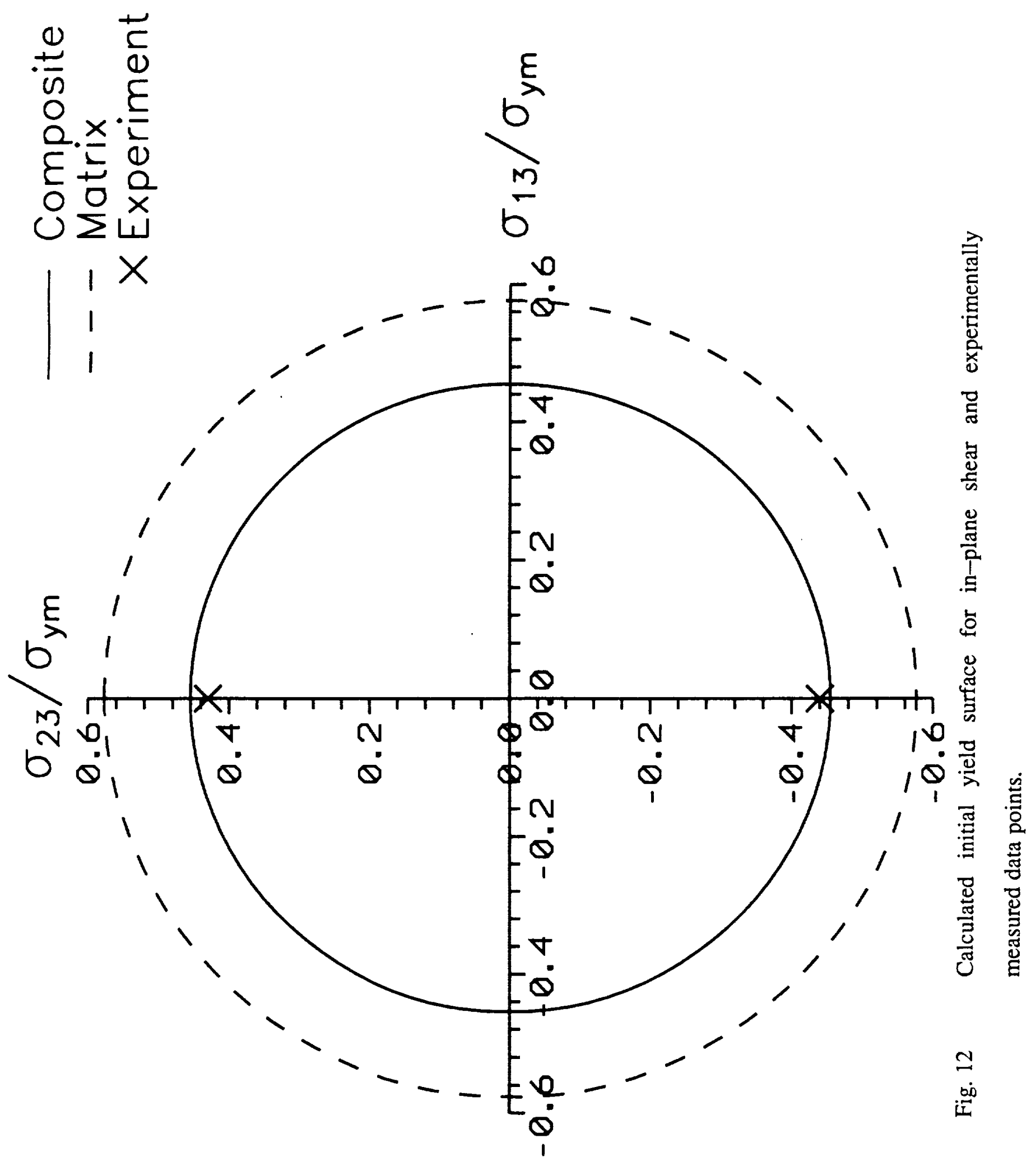




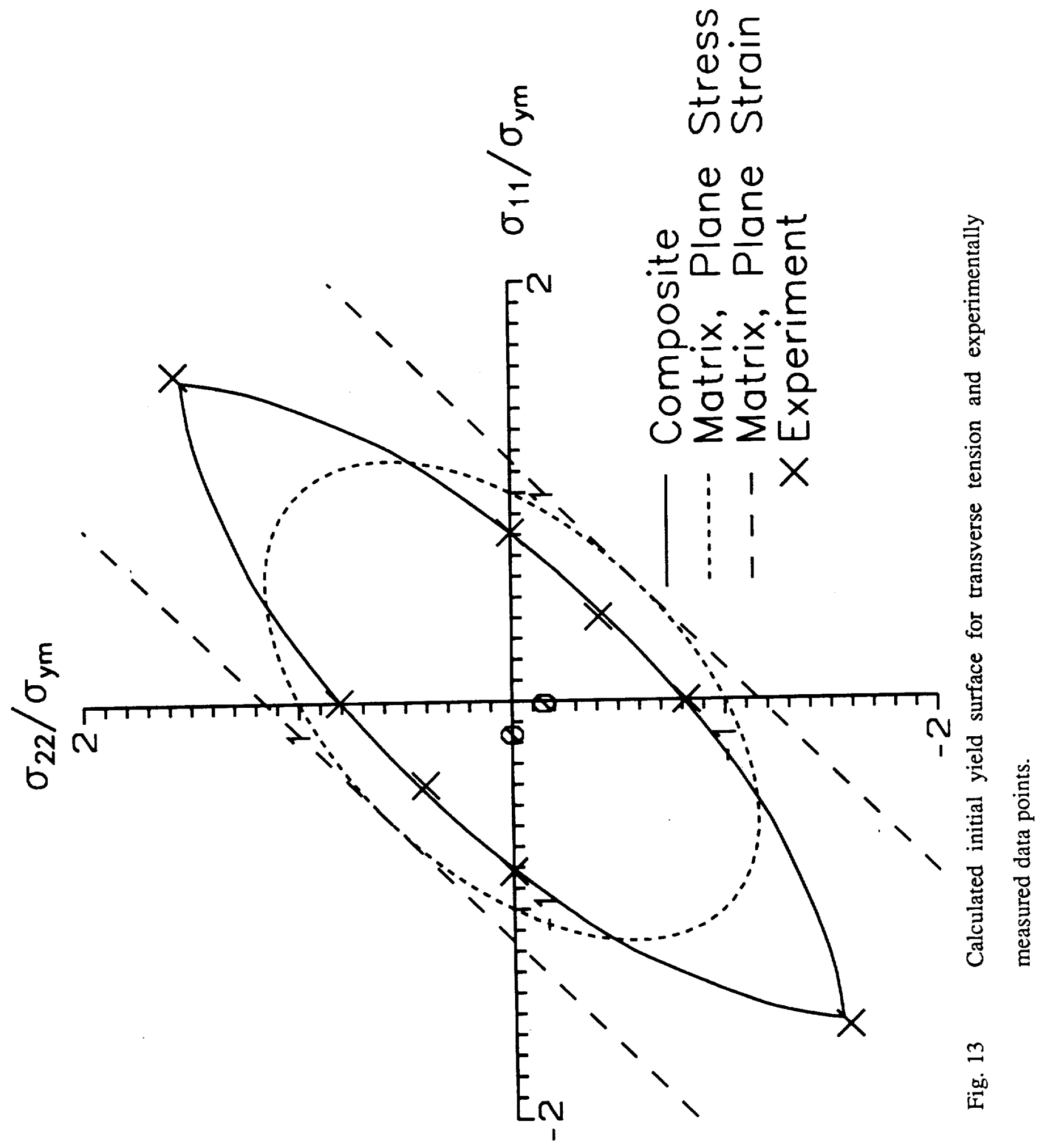




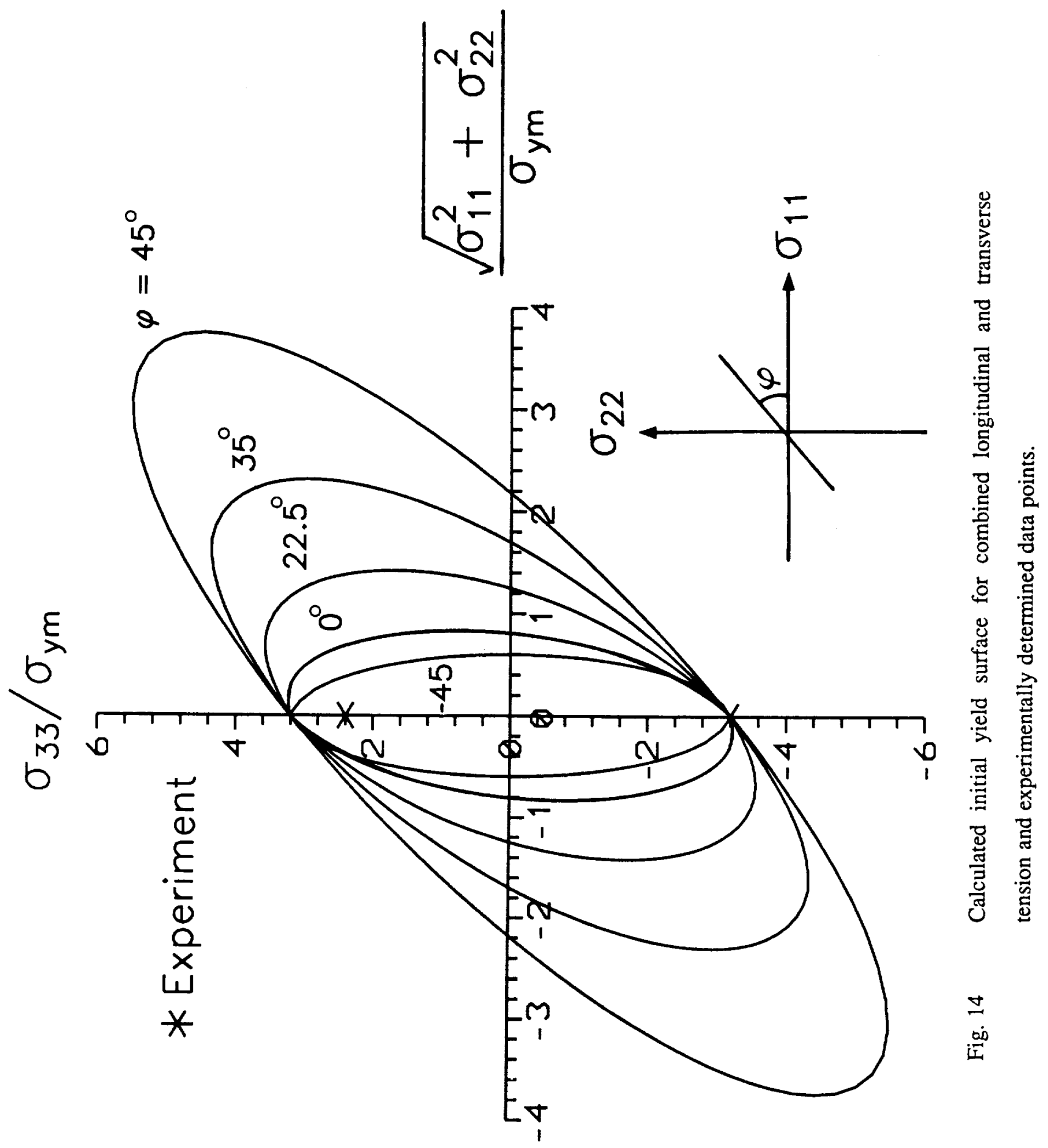




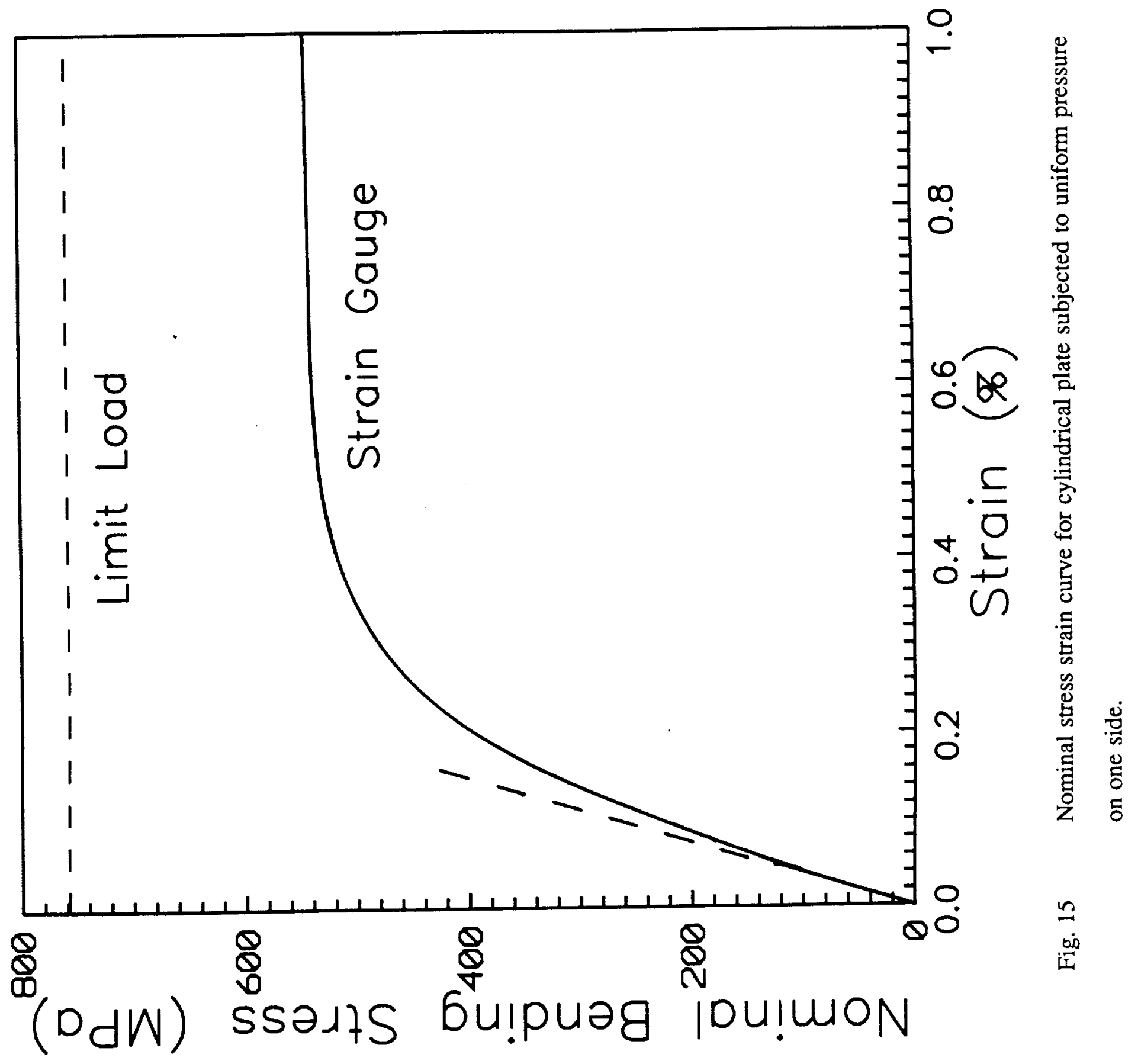




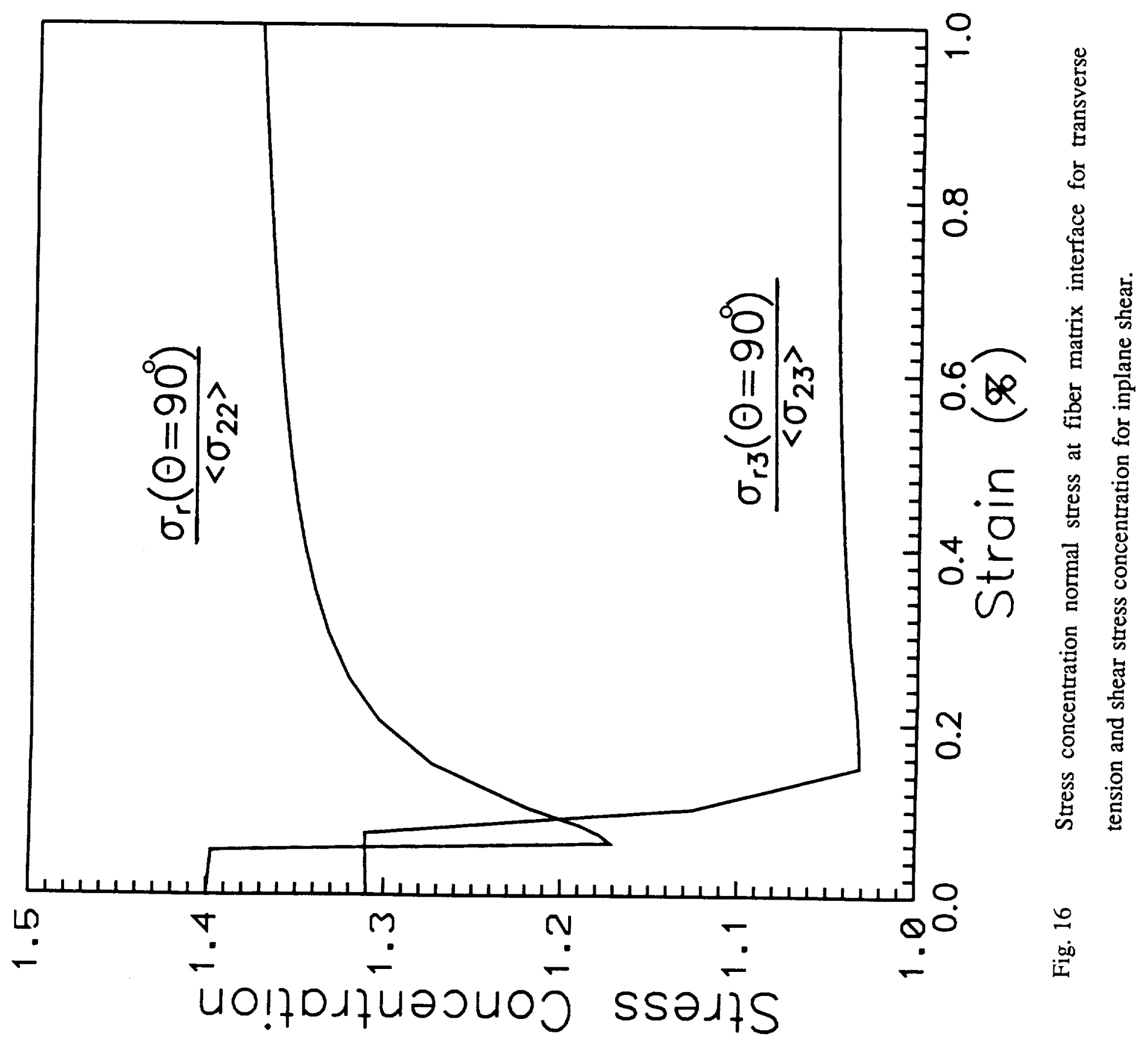




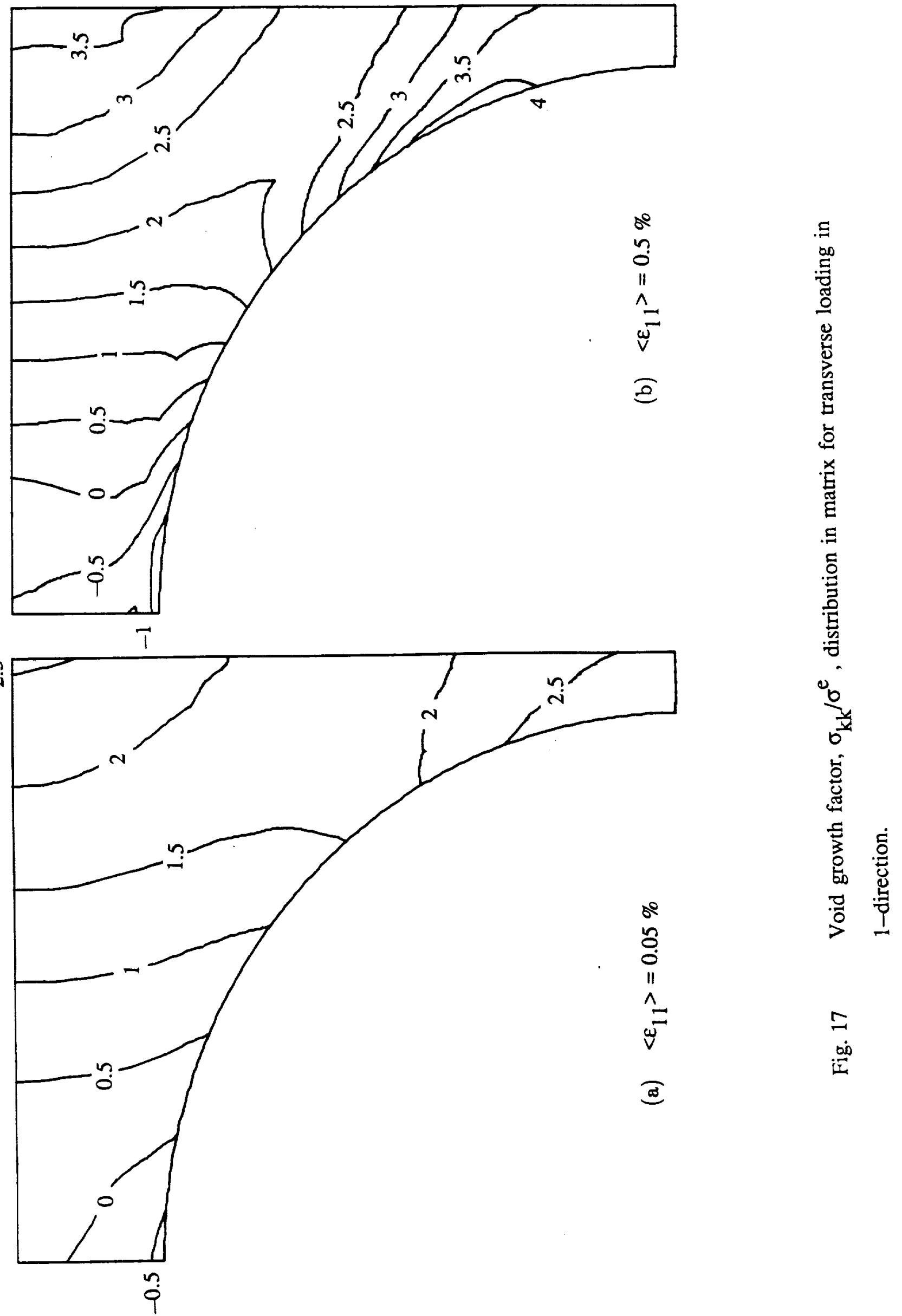




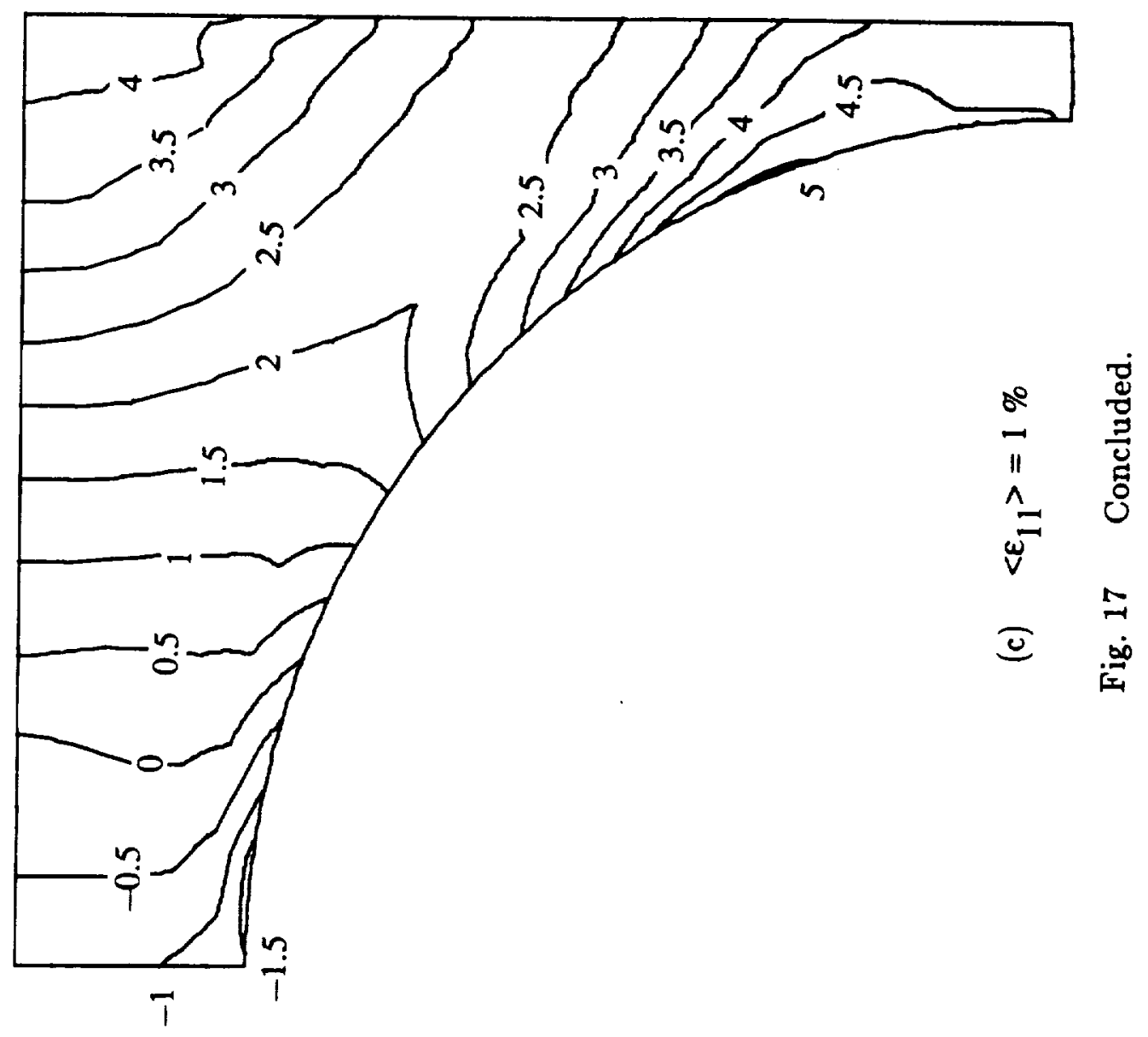




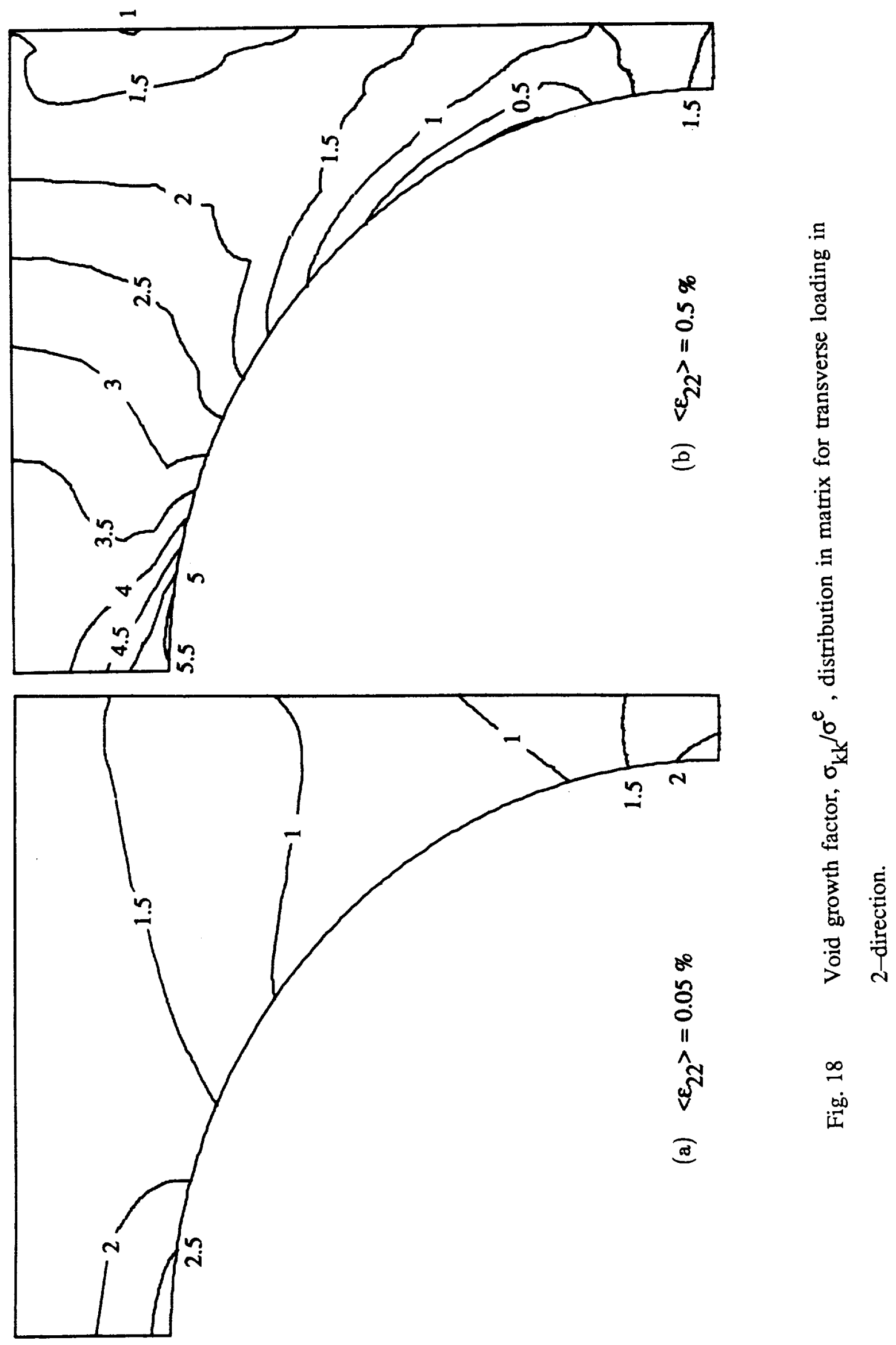




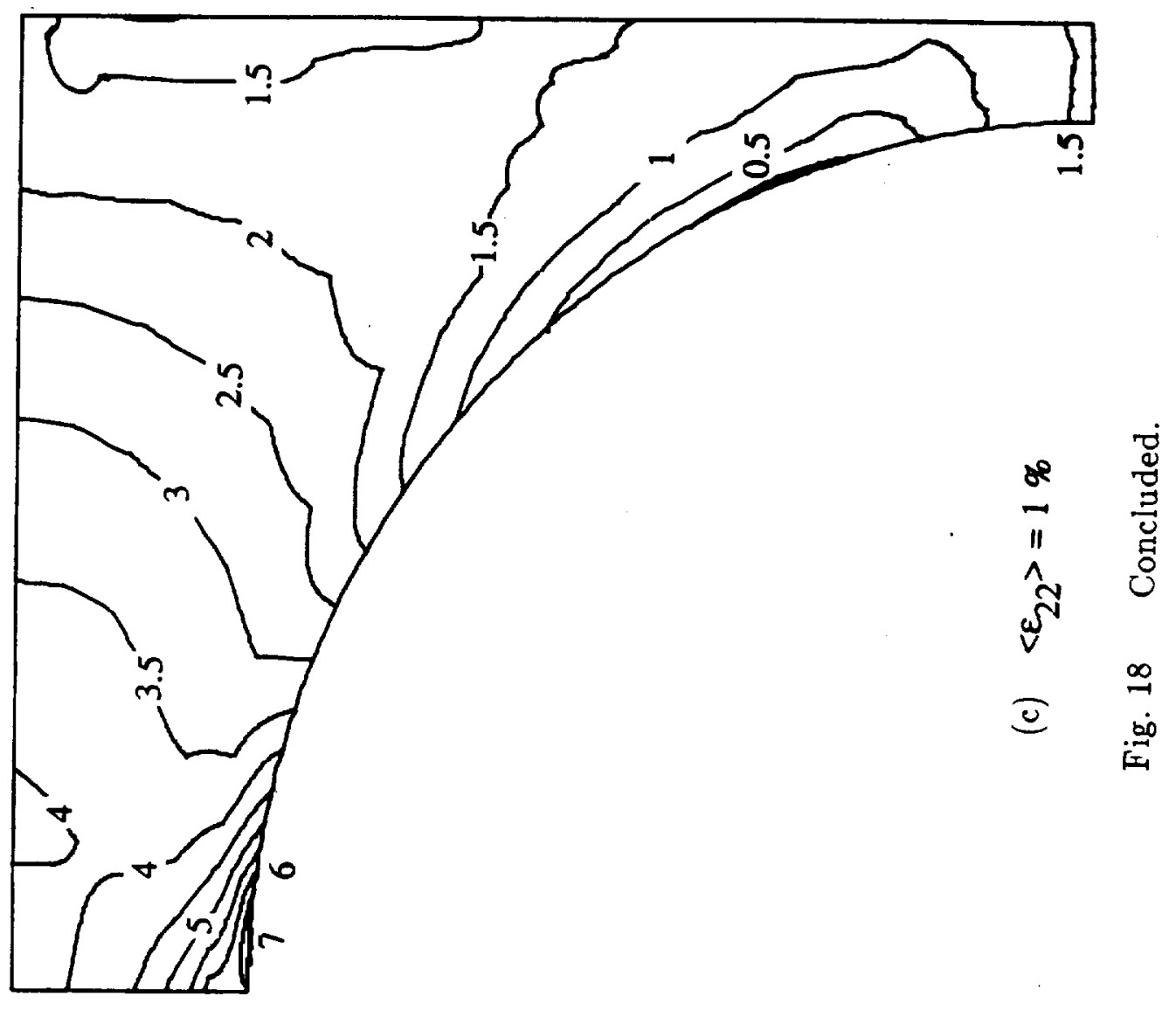




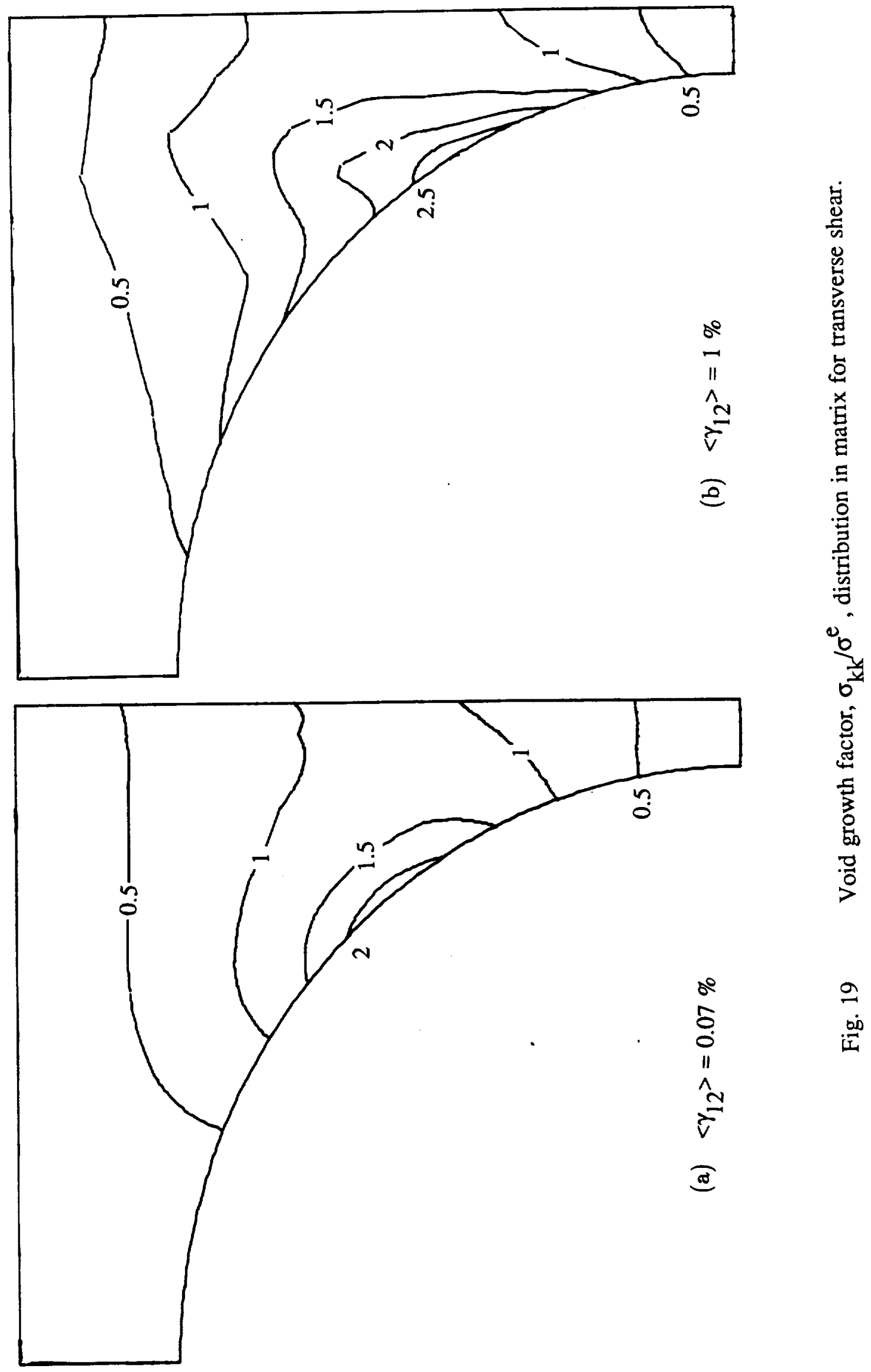




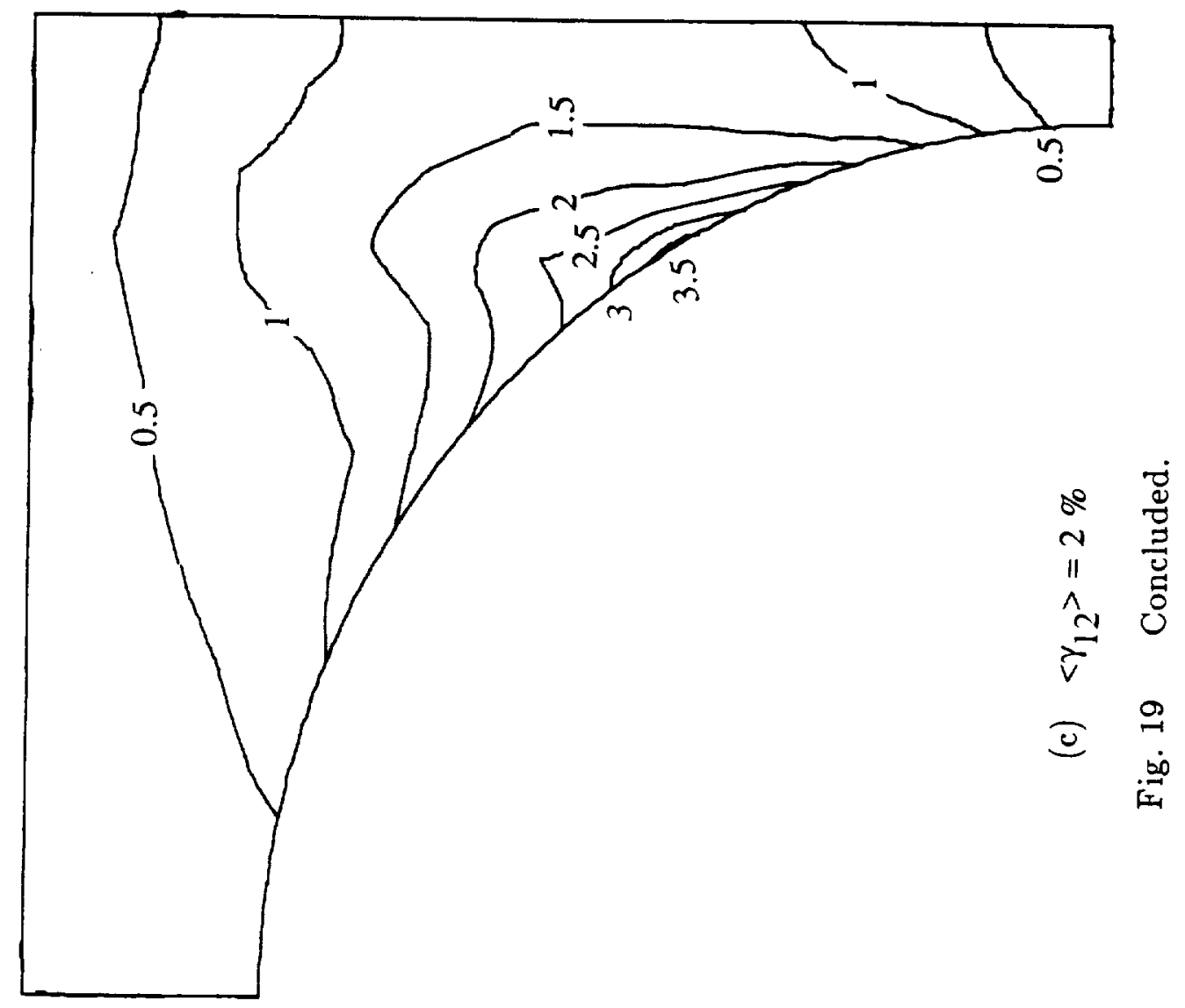




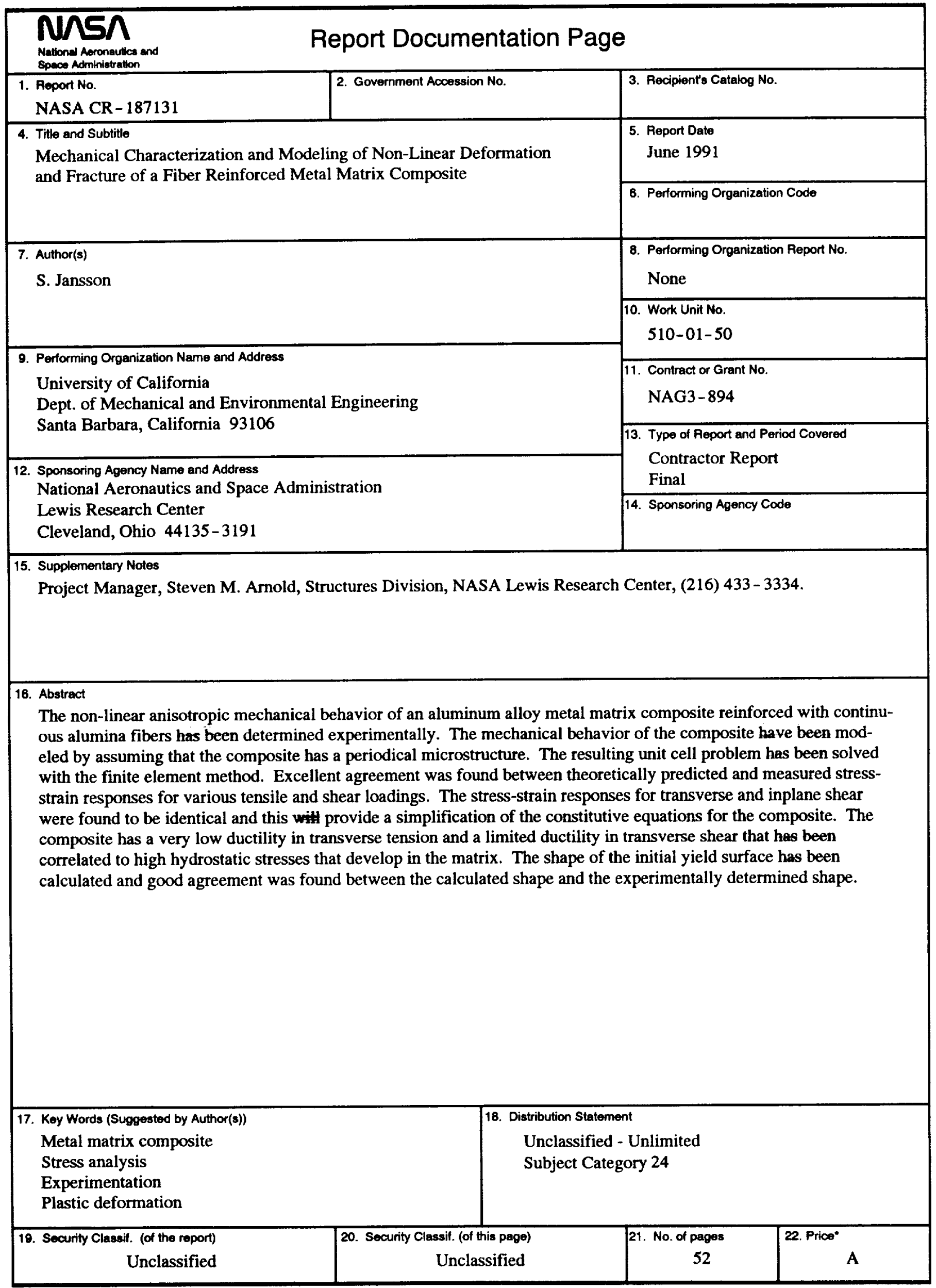

